\title{
The global medial structure of regions in $\mathbb{R}^{3}$
}

\author{
JAMES DAMON
}

For compact regions $\Omega$ in $\mathbb{R}^{3}$ with generic smooth boundary $\mathcal{B}$, we consider geometric properties of $\Omega$ which lie midway between their topology and geometry and can be summarized by the term "geometric complexity". The "geometric complexity" of $\Omega$ is captured by its Blum medial axis $M$, which is a Whitney stratified set whose local structure at each point is given by specific standard local types.

We classify the geometric complexity by giving a structure theorem for the Blum medial axis $M$. We do so by first giving an algorithm for decomposing $M$ using the local types into "irreducible components" and then representing each medial component as obtained by attaching surfaces with boundaries to 4 -valent graphs. The two stages are described by a two level extended graph structure. The top level describes a simplified form of the attaching of the irreducible medial components to each other, and the second level extended graph structure for each irreducible component specifies how to construct the component.

We further use the data associated to the extended graph structures to express topological invariants of $\Omega$ such as the homology and fundamental group in terms of the singular invariants of $M$ defined using the local standard types and the extended graph structures. Using the classification, we characterize contractible regions in terms of the extended graph structures and the associated data.

57N80; 68U05, 53A05, 55P55

\section{Introduction}

We consider a compact region $\Omega \subset \mathbb{R}^{3}$ with generic smooth boundary $\mathcal{B}$. We are interested in geometric properties of $\Omega$ and $\mathcal{B}$ which lie midway between their topology and geometry and can be summarized by the term "geometric complexity". Our goal in this paper is first to give a structure theorem for the "geometric complexity" of such regions. Second, we directly relate this structure to the topology of the region and deduce how the topology places restrictions on the geometric complexity and how the structure capturing the geometric complexity determines the topology of the region. 
To explain what we mean by geometric complexity, we first consider $\mathbb{R}^{2}$. If $\mathcal{B}$ is a simple closed curve as in Figure 1 (a), then by the Jordan Curve and Schoenflies Theorems, $\Omega$ in Figure 1 (b) is topologically a $2-$ disk, and hence contractible. Hence, the region in Figure 1 (b) is topologically simple; however, it has considerable "geometric complexity". It is this geometric complexity which is important for understanding shape features for both 2 and 3 dimensional objects in a number of areas such as computer and medical imaging, biology, etc.

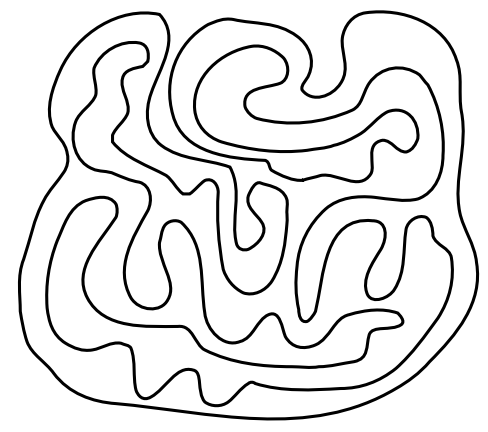

(a)

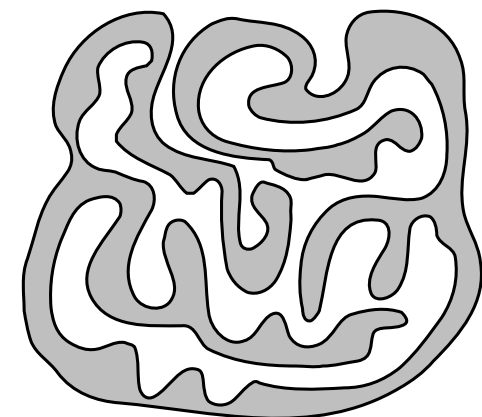

(b)

Figure 1: Simple closed curve in (a) bounding a contractible region in $\mathbb{R}^{2}$ in (b)

This complexity is not captured by traditional geometrical invariants such as the local curvature of $\mathcal{B}$ nor by global geometric invariants given by integrals over $\mathcal{B}$ or $\Omega$. Nor do traditional results such as the Riemann mapping theorem, which provides the existence of a conformal diffeomorphism of $D^{2}$ with $\Omega$, provide a comparison of the geometric complexity of $\Omega$ with $D^{2}$. For 2 -dimensional contractible regions, a theorem of Grayson [13], building on the combined work of Gage and Hamilton [9; 10], shows that under curvature flow the boundary $\mathcal{B}$ evolves so the fingers of the region shrink and the region ultimately simplifies and shrinks to a convex region which contracts to a "round point". Throughout this evolution, the boundary of the region remains smooth. The order of shrinking and disappearing of subregions provides a model of the geometric complexity of the region. Unfortunately, this approach already fails in $\mathbb{R}^{3}$, where the corresponding mean curvature flow may develop singularities, as in the case of the "dumbell" surface found by Grayson (also see eg Sethian [18]).

The structure theorem for the "geometric complexity" which we give is based on the global structure of the "Blum medial axis" $M$. The medial axis is a singular space which encodes both the topology and geometry of the region (see Figure 2). It is defined in all dimensions and has multiple descriptions including locus of centers of spheres in $\Omega$ which are tangent to $\mathcal{B}$ at two or more points (or have a degenerate tangency) as in Blum and Nagel [1], the shock set for the eikonal/ "grassfire" flow as 
in Kimia, Tannenbaum and Zucker [14], which is also a geometric flow on $\Omega$, and the Maxwell set for the family of distance to the boundary functions as in Mather [16]. It has alternately been called the central set by Yomdin [20], and has as an analogue the cut-locus for regions without conjugate points in Riemannian manifolds.

The multiple descriptions allow its local structure to be explicitly determined for regions in $\mathbb{R}^{n+1}$ with generic smooth boundaries: $M$ is an $n$-dimensional Whitney stratified set (by Mather [16]) which is a strong deformation retract of $\Omega$. The local structure of $M$ is given by a specific list of local models, by Blum and Nagel for $n=1$, by Yomdin [20] for $n \leq 3$ (where it is called the central set) and Mather [16] for $n \leq 6$, and is given a precise singularity theoretic geometrical description by Giblin [11] for $n=2$. Results of Buchner [4] show the cut locus has analogous properties for regions without conjugate points (and our structure theorem extends to this more general case). Furthermore, by results in $[6 ; 7 ; 8 ; 5]$, we can derive the local, relative, and global geometry of both $\Omega$ and $\mathcal{B}$ in terms of geometric properties defined on $M$.

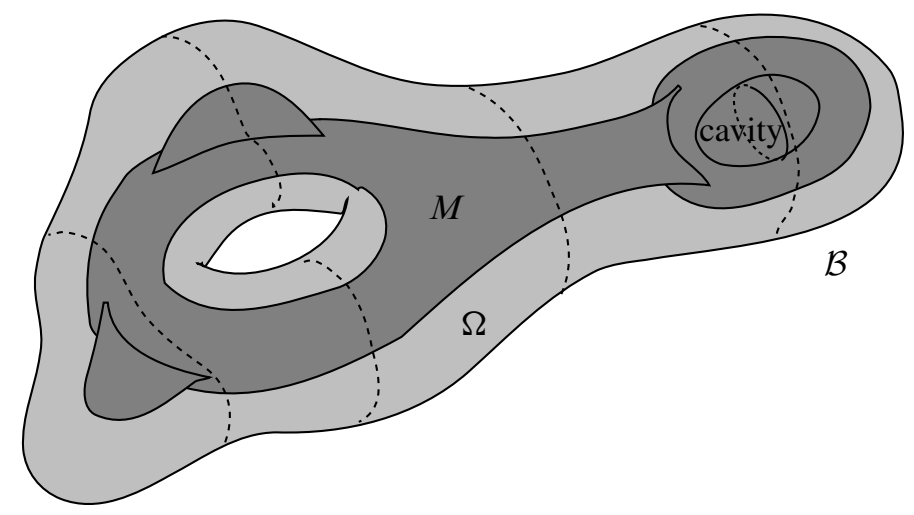

Figure 2: Blum medial axis for a region in $\mathbb{R}^{3}$

For example, for generic compact regions in $\mathbb{R}^{2}, M$ is a 1 -dimensional singular space whose singular points either have $Y$-shaped branching or are end points. This defines a natural graph structure with vertices for the branch and end points and edges representing the curve segments joining these points (see Figure 3). This graph structure encodes the geometric complexity of the region. Furthermore, $\Omega$ is contractible if and only if the graph is a tree. Then the tree structure can be used to contract the region (also for computer imaging a tree structure is a desirable feature, as trees can be searched in polynomial time). A computer scientist Mads Nielsen has asked whether in the case of contractible $\Omega \subset \mathbb{R}^{3}$, the Blum medial axis still has a "tree structure". We answer this question as part of the structure theorem for the medial axis $M$ for generic regions. 

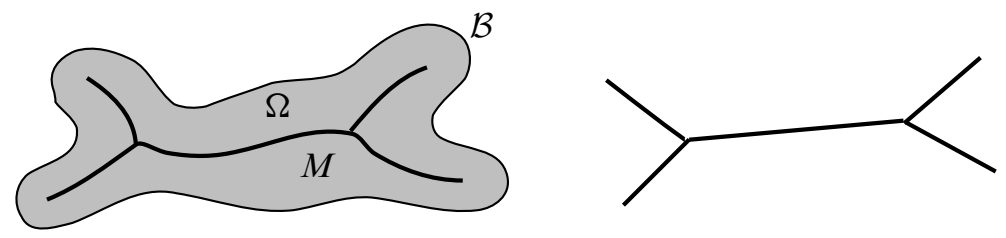

Figure 3: Blum medial axis for a region in $\mathbb{R}^{2}$ with associated graph structure

While the global structure of the medial axis as a Whitney stratified set with specific local singular structure does capture the geometric complexity of $\Omega$, it is insufficient in this raw form to characterize the geometric complexity or to see directly its relation with the topology. This is what the structure theorem accomplishes.

We give a brief overview of the form of the structure theorem (Theorem 4.1).

\section{The structure theorem for general regions}

At the top level we decompose $M$ into "irreducible medial components" $M_{i}$ which are joined to each other along "fin curves". Geometrically this corresponds to taking connected sums of 3-manifolds with boundaries. A simplified form of the attaching is described by a top level directed graph $\Gamma(M)$. We assign vertices representing the $M_{i}$, and directed edges from $M_{i}$ to $M_{j}$ for each edge component of $M_{i}$ attached to $M_{j}$ along a fin curve. This is an "extended graph" in that there may be more than one edge between a pair of vertices or edges from a vertex to itself (see Figure 4). The resulting space obtained by attaching the $M_{i}$ as indicated by the edges of $\Gamma(M)$ is the simplified form $\hat{M}$ of the original $M$. It is homotopy equivalent to $M$ and has the same irreducible components. From the topological perspective, the decomposition is optimal in that the homology and fundamental group of $M$ are decomposed into direct sums, resp. free products, of those for the $M_{i}$. Also, $M$ can be recovered from $\hat{M}$ by certain "sliding operations along fin curves" according to additional data defined from $M$.

At the second level, we describe the structure of each irreducible medial component $M_{i}$ by an extended graph $\Lambda\left(M_{i}\right)$, denoted more simply by $\Lambda_{i}$, as in Figure 5. Here, there is an analogy with the resolution graph of an isolated surface singularity, whose vertices correspond to complex curves, ie compact orientable real surfaces, (with data the genera and self-intersection numbers) and edges indicating transverse intersection. Alternately, we can view the vertices as denoting multiply-punctured real surfaces which are attached to the set of intersection points as indicated by the edges. For the irreducible medial components there is an analogous structure but with several added complications. 


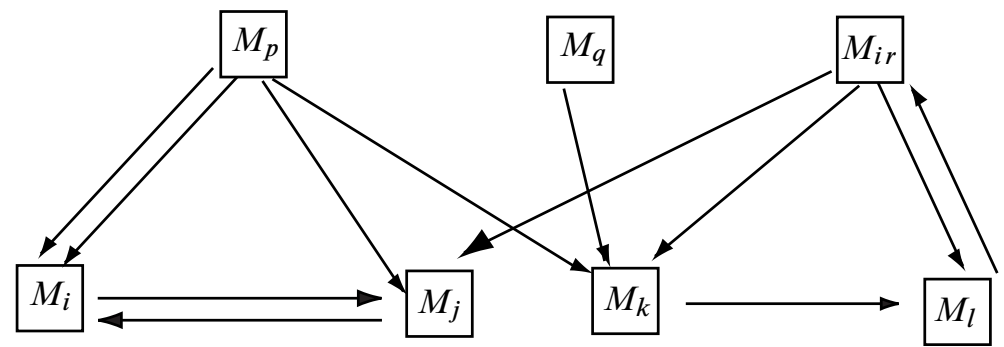

Figure 4: Graph structure given by decomposition into irreducible medial components with edges indicating the attaching along "fin curves"

The extended graph $\Lambda_{i}$ corresponding to an irreducible medial component $M_{i}$ has two types of vertices: " $S$-vertices" which are attached by edges to " $Y$-nodes". The $S$-vertices correspond to the medial sheets $S_{i j}$, which are (closures of) connected components of the set of smooth points of the component $M_{i}$ (which are not in general the connected components of the set of smooth points of $M$ ). They are compact surfaces with boundaries (possibly nonorientable). The $Y$-nodes correspond to connected components $\mathcal{Y}_{i j}$ of the " $Y$-network" $\mathcal{Y}_{i}$ of $M_{i}$. The $Y$-network of $M_{i}$ is the collection of " $Y$-branch curves" together with vertices which are the " 6 -junction points" where 6 sheets of the Blum medial axis come together in a point along 4 $Y$-branch curves (and is described by a 4-valent extended graph). Each edge from a vertex for $S_{i j}$ to a node for $\mathcal{Y}_{i k}$ represents the attaching of the sheet $S_{i j}$ along one of its boundary components to the component $\mathcal{Y}_{i k}$.

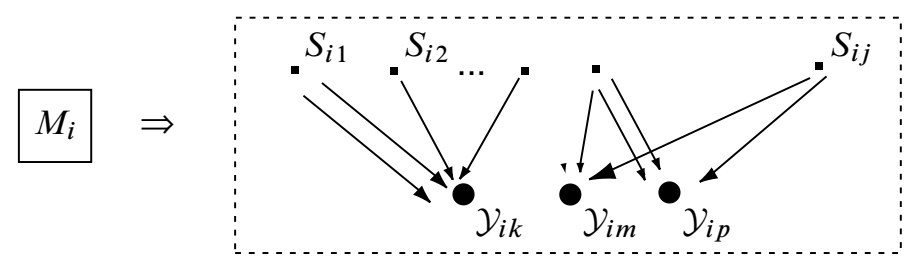

Figure 5: Extended graph structure for irreducible medial component $M_{i}$ : $S$-vertices $\bullet$ representing connected medial sheets $S_{i j}$ joined to $Y$-nodes representing components $\mathcal{Y}_{i k}$ of the " $Y$-network"

There is associated data attached to the vertices and edges of $\Lambda_{i}$; namely, for $S$-vertices the genus, orientability, and number of medial edge curves on $S_{i j}$, for $Y$-nodes the 4-valent extended graph associated to $\mathcal{Y}_{i k}$, and for each edge the attaching data of the boundary component of $S_{i j}$ to $\mathcal{Y}_{i k}$.

From this structure we obtain two alternate ways to view $M_{i}$ : either as obtained by attaching compact surfaces with boundaries along (some of the) boundary components 
or by an associated CW-decomposition. These allow us to relate the graph structures and their associated data for all of the $M_{i}$ to the topological structure of $\Omega$.

Topological invariants of $M$, and hence $\Omega$, are computed by Theorem 3.2 and Theorem 3.4 in terms of $M_{i}$ and $\Gamma(M)$. In turn, those of each $M_{i}$ are expressed in terms of the extended graph $\Lambda_{i}$, and the associated data. This data includes the singular invariants of each $M_{i}$ such as the numbers of 6-junction points, medial edge curves, components of $\mathcal{Y}_{i}$, the genera of $S_{i j}$, etc. From this we define a single "algebraic attaching map" for $M_{i}$. From this data, we compute in Theorem 3.2 the topological invariants of $M_{i}$ such as homology groups and Euler characteristic. We then deduce in Theorem 3.4 the corresponding invariants for $M$ and hence, $\Omega$. Also, in Theorem 7.4 we give a presentation of $\pi_{1}\left(M_{i}\right)$ in terms of the associated data for $\Lambda_{i}$. These provide topological bounds on the geometric complexity of $\Omega$.

The answer to Nielsen's question turns out in general to be no provided we wish to classify the medial axis up to homeomorphism. The tree has to be replaced by an Algorithm we give in Section 1 for decomposing the medial axis into the irreducible components $M_{i}$. The data of the algorithm requires more than just a graph showing which medial components are attached to which. However, the simplified version $\widehat{M}$, which sacrifices some of the detail of the attachings, is described by an extended graph $\Gamma(M)$, and for contractible regions $\Gamma(M)$ is a tree. Then the special form which the structure theorem takes for contractible regions in $\mathbb{R}^{3}$ (Theorem 5.2) gives a complete characterization of contractible regions by the following conditions: the extended graphs $\Gamma(M)$ and the $\Lambda_{i}$ are trees, the medial sheets have genus 0 (and so are topological 2 -disks with a finite number of holes) with at most one boundary curve representing a medial edge curve, a numerical "Euler relation" is satisfied which involves the basic medial invariants, and a fundamental group condition holds (Condition 5.1) which involves a space formed from $\mathcal{Y}_{i}$ and $\Lambda_{i}$.

The author is especially grateful to Mads Nielsen for initially raising the question about the tree structure for the contractible case, which led this investigator to these questions and results.

Acknowledgements This research was partially supported by grants from the National Science Foundation DMS-0405947 and CCR-0310546 and a grant from DARPA.

\section{Decomposition into irreducible medial components}

\section{Generic local structure of Blum medial axis}

We consider a region $\Omega \subset \mathbb{R}^{3}$ with generic smooth boundary $\mathcal{B}$ and Blum medial axis $M$. Then by Mather [16], $M$ can be viewed as the Maxwell set for the family of 
distance functions on $\mathcal{B}$; hence, for generic $\mathcal{B}$, it is a 2 -dimensional Whitney stratified set. Also, the generic local structure has one of the following local forms in Figure 6 (see eg [11], where Giblin gives a very explicit geometric description).

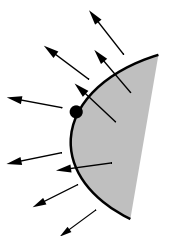

(a) edge

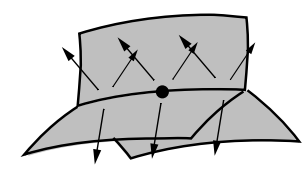

(b) Y-branching

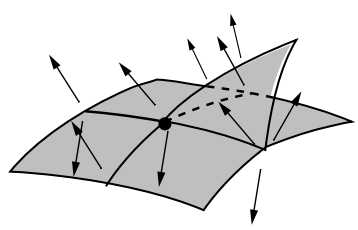

(c) fin creation point

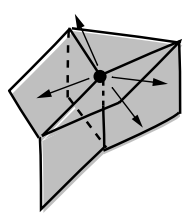

(d) "6-junction"

Figure 6: Local generic structure for Blum medial axes in $\mathbb{R}^{3}$ and the associated radial vector fields to points of tangency on the boundary

Then $M$ consists of the following: (i) smooth connected (2-dimensional) strata; 1-dimensional strata consisting of (ii) $Y$-junction curves along which three strata meet in a $Y$-branching pattern and (iii) edge curves consisting of edge points of $M$; 0 -dimensional strata consisting of (iv) fin points and (v) 6 -junction points, where six medial sheets meet along with $4 Y$-junction curves. Connected components of $Y$-junction curves end either at fin points or 6 -junction points; while edge curves only end at fin points. We refer to the union of $Y$-junction curves, fin points, and 6-junction points as the initial $Y$-network $\mathcal{Y}$.

In the simplest form we can view the medial axis as formed by attaching the connected 2dimensional smooth strata to the 1-complex formed from the $Y$-branch and medial edge curves and the fin and 6 -junction points. In fact, this approach misses a considerable amount of global structure for subspaces of $M$. To identify this larger structure we will decompose $M$ into "irreducible medial components" $M_{i}$ by "cutting $M$ along fin curves". Then we further decompose the irreducible medial components $M_{i}$ by representing them as obtained by attaching smooth medial sheets to the resulting $Y_{-}$ network formed from the union of $Y$-junction curves and 6-junction points in $M_{i}$. From this we will ultimately give a $\mathrm{CW}$-decomposition to compute the topological invariants of $M$.

\section{Cutting the medial axis along fin curves}

In order to proceed, we first explain how we cut along fin curves.

Suppose we have on the Blum medial axis a fin point $x_{0}$. Then near $x_{0}$ we can distinguish the "fin sheet" which is the sheet that contains a medial axis edge curve ending at $x_{0}$. We can begin following the $Y$-branch curve from $x_{0}$, while keeping track of the fin sheet which remains a connected sheet as we move along the curve. 
Eventually one of two things must happen: either we reach a 6 -junction point or another fin point.

First, if the $Y$-branch curve meets a 6 -junction point, then because the fin sheet is locally connected near the 6 -junction point, we can follow the edge of the sheet as it continues through the 6 -junction point. After the 6 -junction point we have identified both the corresponding continuation of the $Y$-branch curve, and the sheet. We can do this for each 6-junction point it encounters. As $M$ is compact, eventually the $Y$-branch curve must meet another fin point. We shall refer to the edge of the sheet from one fin point to the other as a fin curve.

At the end of the fin curve, what was identified as the fin sheet (close to the $Y$-branch curve) from the beginning may or may not be the fin sheet for the end point. If the sheet is a fin sheet at both ends, then we refer to the fin curve as being "essential", while if it is only a fin sheet at one end, then we refer to the fin curve as "inessential", (later discussion will explain the reason for these labels). Examples of these are shown in Figure 7.

(a)

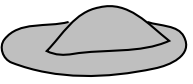

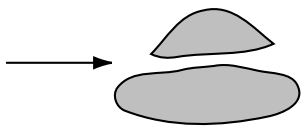

(b)

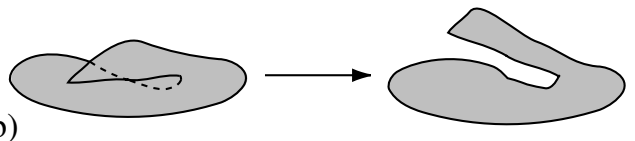

Figure 7: Two possibilities for fin curves on a medial sheet and the results of cutting along the fin sheets: (a) essential fin curve (b) inessential fin curve

An example of a region containing an inessential fin curve is given in Figure 8 and might be called a "Mobius board", a surf board but with a "Mobius band" twist.

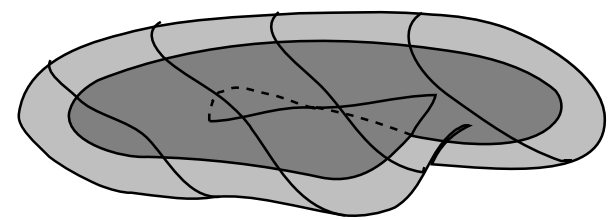

Figure 8: "Mobius board" with a "inessential fin curve"

Then to cut along a fin curve beginning from a fin point $x_{0}$, we locally disconnect the fin sheet from the direction of $x_{0}$ by locally adding a point of closure to the fin sheet for each point of closure on the fin curve (as followed from $x_{0}$ ). We must take care as a point on the fin curve can locally be a point of closure for more than one part of the sheet. After this step, locally at an added closure point, the fin sheet is now a surface with piecewise smooth boundary; see Figure 7. If the fin curve is essential, then the fin 
sheet is now locally disconnected from the remaining two sheets still attached along the $Y$-junction curve. While if the fin curve is inessential, the fin sheet is still attached at the other fin point to the remaining two sheets; again see Figure 7 (b).

Then we can take the two remaining sheets still attached along that fin curve and smooth them to form a smooth sheet along the curve, with former 6-junction points on the fin curve becoming $Y$-branch points (for another $Y$-branch curve).

After having cut along the fin curves as described, the former points on the fin curve have become altered as follows:

(1) Fin points become points of an edge of the fin sheet.

(2) A former $Y$-branch point becomes a closure point on an edge of a fin sheet.

(3) A former 6-junction point become a (topological) $Y$-network point.

(4) A $Y$-branch point on a base sheet becomes a (topological) 2-manifold point of that sheet.

Because of (4) above, the end result depends upon a further distinction for essential fin curves. A type-1 essential fin curve will be one which only intersects other essential fin curves at 6-junction points; otherwise, it shares a segment of $Y$-branch curve with another essential fin curve, and it will be type-2 essential fin curve (see eg Figure 9). If we cut along a type- 1 essential fin curve, then the fin sheet becomes disconnected from the other sheets (at least along the curve) and this does not alter any other essential fin curve. If we cut along a type-2 essential fin curve, then it will alter the structure of the other essential fin curves sharing a segment of $Y$-branch curve with it. Hence, we can prescribe the following algorithm for decomposing the medial axis.

\section{Algorithm for decomposing medial axis into irreducible components}

(1) Identify all type-1 essential fin curves and systematically cut along type-1 essential fin curves (it does not matter which order we choose as cutting along one does not alter the fin properties of another).

(2) After cutting along all type-1 essential fin curves, we may change certain inessential fin curves to type-1 essential ones. If so return to step (1).

(3) There only remain type-2 essential fin curves and inessential fin curves. Choose an essential fin curve and cut along it. If a type-1 essential fin curve is created, return to step (1). Otherwise, repeat this step until no essential fin curves remain.

(4) When there are no other essential fin curves, choose an inessential fin curve which crosses a 6 -junction point, and cut it from one side until we cut across one 6-junction point. 
(5) Check whether we have created an essential fin curve. If so then we cut along it, and repeat the earlier steps (1)-(3).

(6) If no essential fin curve is created, then we repeat step (3) until there are only inessential fin curves which do not cross 6 -junction points.

(7) Finally we can cut each such remaining inessential fin curve, producing part of a smooth sheet as in Figure 7 (b).

(8) The remaining connected pieces are the "irreducible medial components" $M_{i}$ of $M$.

Remark 1.1 The distinct connected pieces created following steps (1) and (2) are intrinsic to $M$; while those created using steps (3) and (4) are not because choices are involved. Which choices are made typically depends on the given situation and the importance we subjectively assign to how sheets are attached.

Example 1.2 In Figure 9(a), we have a contractible medial axis with a pair of type-2 essential fin curves. Depending on which essential fin curve we choose, 1-2 or 3-4, we choose to cut along in step (3), we obtain either (b) or (c), which leads to different attachings (and hence top level graph) for the irreducible medial components. An alternate possibility would be to cut each fin sheet along the fin curves and view them as being attached partially along the edge of a fourth sheet. Again, the exact geometric form of $M$ may suggest one choice being preferred over the others.

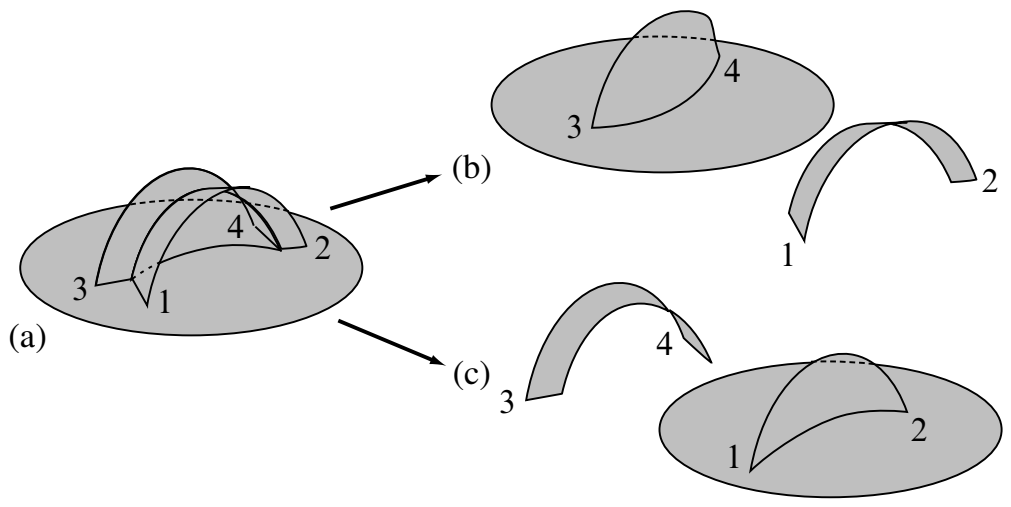

Figure 9: Nonuniqueness of medial decomposition resulting from type2 essential fin curves: (a) is a contractible medial axis with only type-2 essential fin curves, and (b) and (c) illustrate the results from cutting along the fin curves 1-2 or 3-4. 
Example 1.3 In Figure 10(a), we have a contractible medial axis with 10 fin points $1-10$, and all fin curves are inessential. Depending on how we choose cuts in step (4) of the algorithm, we can end up with 1, 2, or 3 irreducible medial components.

If we cut from 4 through the first 6-junction point, then 3-6 becomes an essential fin curve, and we cut away the fin sheet $M_{1}$ as in Figure 10 (b). Then further cutting from 8 through the first 6-junction point, we create another essential fin curve 2-9. Cutting along it creates a second fin sheet $M_{2}$. The remaining inessential fin curves 1-4, 5-7, and 8-10 can be contracted to points on edges of the third sheet $M_{3}$. Each of these 3 medial sheets are then irreducible components.

Alternatively, after the first cut, we could have instead cut from 9, and then from 4 again, and then only inessential fin curves remain without 6 -junction points, so they contract to a second sheet, and we only obtain two irreducible components. Thirdly, we could have begun cutting from 7 , then 8 , and then 4 twice and we would obtain only a single medial sheet with inessential fin curves, leading to a single irreducible component.

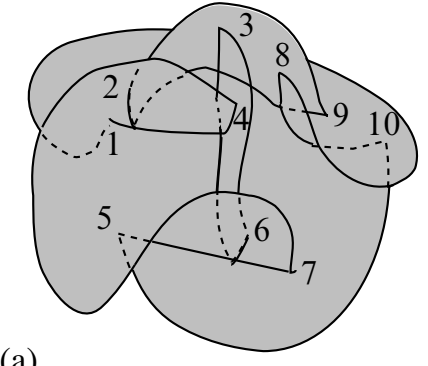

(a) (b)

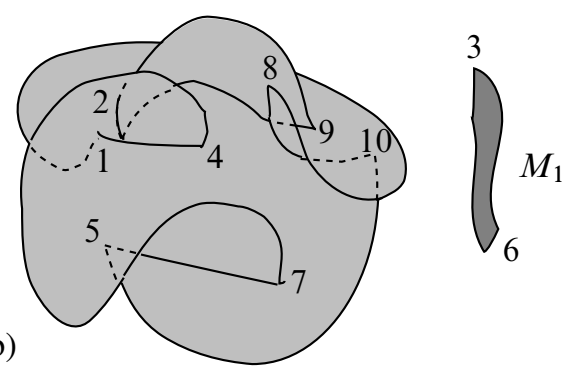

Figure 10: Nonuniqueness of medial decomposition resulting from inessential fin curves: (a) is a contractible medial axis with only inessential fin curves, and (b) illustrates the cutting of irreducible medial component $M_{1}$ after first cutting from fin point 4 .

\section{Constructing the medial axis by attaching irreducible medial components along fin curves}

To reverse the algorithm and reconstruct $M$ from the $M_{i}$ requires the following additional attaching data:

(1) the list of which segments of medial edge curves which will be attached as either essential or inessential fin curves

(2) the order in which the sheets will be attached 
(3) the curve at the $j$-th stage to which the attaching will be made as a fin curve to obtain the $(j+1)-$ st stage.

The curves in step (3) may cross multiple components, and need only be well- defined up to isotopy; however, this isotopy is for the space obtained at the $j$-th stage, whose singular set must be preserved by the isotopy. Hence for example, in Figure 11, even though the medial axis is contractible, if the attaching curve were on the same side of the two holes then the resulting spaces would not be homeomorphic.

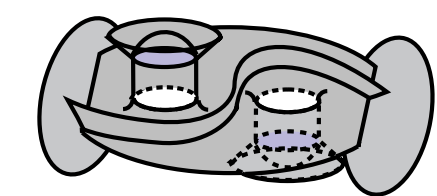

(a)

Figure 11: (a) A contractible medial axis, with attaching of component $M_{4}$ to multiple components $M_{1}, M_{2}, M_{3}$ for (a) (b)

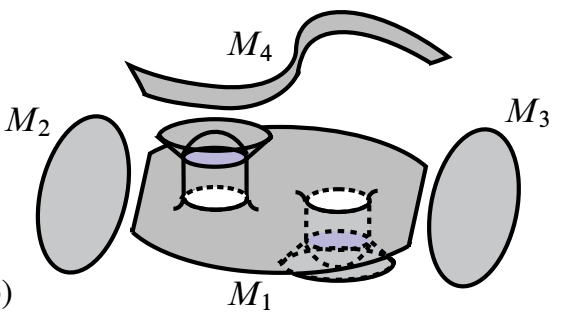

\section{Extended graph structure and medial decomposition}

We next turn to a simplified version of $M$ using the same irreducible medial components, but with simplified attaching. The resulting simplified version of $M$, which we denote by $\widehat{M}$, will still be homotopy equivalent to $M$. To define $\widehat{M}$, we consider an alternate way to understand the topological effects of attaching along a fin curve $\gamma$. Instead, we isotope the attaching map along the support of the original $\gamma$, by sliding and shrinking so it now becomes a fin curve $\gamma^{\prime}$ which no longer passes through any 6 -junction points (which have disappeared after the isotopy as in Figure 12).

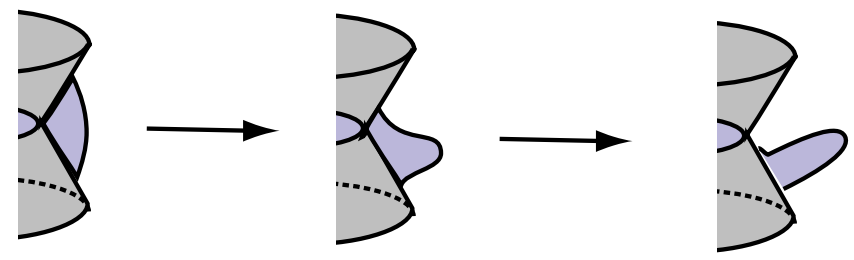

Figure 12: Isotoping a fin curve so it misses 6-junction points 
We can achieve this by replacing $M$ by a subcomplex which is a strong deformation retract, and only differs from $M$ in a small neighborhood of the fin curve.

Lemma 2.1 (Isotopy lemma for fin curves) Let $\gamma$ be a fin curve in $M$. Then there is a Whitney stratified set $M^{\prime} \subset M$, which is a strong deformation retract of $M$ and which only differs from $M$ in a given neighborhood of $\gamma$. Furthermore, in that neighborhood, $\gamma$ has been replaced by a fin curve $\gamma^{\prime}$ which does not meet a 6 -junction point.

Proof We obtain $M^{\prime}$ as the result of a series of deformation retractions $M \supset M^{(1)} \supset$ $M^{(2)} \supset \cdots \supset M^{(k)}=M^{\prime}$. Here $M^{(j)} \supset M^{(j+1)}$ corresponds to either moving the fin point along a $Y$-branch curve so it is in a neighborhood of a 6 -junction point where it has the normal form as in Figure 13 (a), or when $M^{(j)}$ already has this form then $M^{(j+1)}$ is a deformation retraction across a 6 -junction point as in Figure $13(\mathrm{~b})$.

In the first case we may construct the deformation because $M$ is analytically trivial along a $Y$-branch curve and so analytically a product in a neighborhood of a compact segment of a $Y$-branch curve. In the second case, we may use the normal form for 6 -junction points to deform along the shown region.

(a)

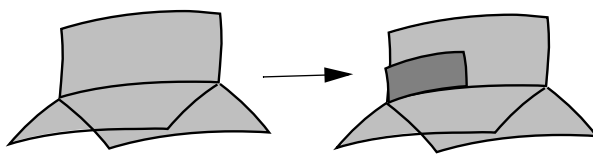

(b)

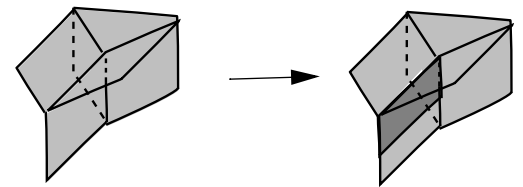

Figure 13: (a) Deformation retraction along a $Y$-branch curve. $\quad$ (b) Deformation retraction across a 6 -junction point. In both cases the deformation is across the dark region.

We view this process as sliding and shrinking the fin curve along its support, across 6-junction points until the fin curve no longer crosses 6-junction points. Also, after isotoping the fin curves, the resulting spaces are still homotopy equivalent.

After first isotoping the fin curves, we can more clearly see the topological effect of cutting along fin curves.

Lemma 2.2 In the preceding situation, let $\gamma^{\prime}$ be a fin curve which does not meet a 6 -junction point. There are two possibilities.

(1) Suppose the fin curve $\gamma^{\prime}$ is essential, with fin sheet $S_{i}$ at both ends. Then when we cut $S_{i}$ along $\gamma^{\prime}$, the sheet locally becomes disconnected from the remaining sheets of the fin curve as in Figure 7 (a). 
(2) If instead the fin curve $\gamma^{\prime}$ is inessential, then we cut all three sheets along the fin curve; we obtain a single sheet with boundary edge containing the fin curve as in Figure 7 (b). This may be alternately obtained by shrinking the fin curve to a point.

Proof In the essential case, by the triviality of $M$ along $Y$-branch sheets, the fin sheet remains a single sheet along the fin curve. When we cut it we disconnect it from the base sheet as in Figure 7 (a). By contrast, in the inessential case when we cut the fin sheet, the edge curve continues along the cut fin curve, joining up with another edge curve again. Hence, the fin points become edge points for a single edge curve.

As an alternate way to view the process, the fin sheet for one fin point becomes part of the base sheet. Then we may follow a path around the second fin point to end up on the base sheet for the first fin point. Then we may continue the curve around the first fin point to be on the sheet which becomes the fin sheet for the second fin point. If we extend these curves so they intersect the edge curve, then together they form a closed curve, so that when the fin curve is contracted to a point we obtain a 2-disk, one edge of which is the edge curve.

Remark 2.3 This Lemma contrasts cutting along essential versus inessential fin curves. When we cut along the essential fin curve we disconnect the fin sheet from the base sheet, causing a change in homotopy type as in Figure 7 (a). By contrast, for inessential fin curves, when we contract the fin curve to a point $p$, the sheet then has an edge curve which passes through $p$. In this case, the "fin curve" can be eliminated, and the pair of fin points cancelled, without any change in the homotopy type of $M$.

Now beginning with a Blum medial axis, we can repeat the Algorithm given in Section 1, except at each step, instead of cutting along the fin curve, by the Isotopy Lemma for Fin Curves, we slide the fin sheet along the fin curve. We pass all of the 6 -junction points (which then become $Y$-branch points), until the fin curve lies on a single smooth sheet. Then by Lemma 2.2 (b), we may contract the inessential fin curves eliminating their fin points. The resulting space with these simplified attachings is $\hat{M}$. Finally we cut the essential fin curves using Lemma 2.2 (b). The resulting connected components $M_{i}$ are again the irreducible medial components of $M$.

The initial $Y$-network has been altered by the removal of the fin curves to yield the $Y$-network $\mathcal{Y}$. Then $\mathcal{Y}=\bigcup_{i} \mathcal{Y}_{i}$, where each $\mathcal{Y}_{i}$ is the resulting $Y$-network for $M_{i}$. Then $\hat{M}$ is obtained from the irreducible medial components by attaching their edges which came from essential fin curves to the isotoped positions in some $M_{j}$. There is some ambiguity in this construction in addition to that coming from the algorithm. We make a choice of the medial sheet that the fin curve passes through. However, the irreducible medial components and the homotopy type of $\hat{M}$ will remain the same. 
This gives rise to a "top level directed extended graph" $\Gamma(M)$ whose vertices correspond to the $M_{i}$, with an edge going from $M_{i}$ to $M_{j}$ for each segment of an edge curve of $M_{i}$ attached to $M_{j}$ along a fin curve.

There is the following relation between the topology of the full medial axis $M$ and that of the irreducible medial components $\left\{M_{i}\right\}_{i=1}^{r}$ and the top level extended graph $\Gamma(M)$.

Proposition 2.4 In the preceding situation

(1) for any coefficient group $G$,

$$
\begin{aligned}
& H_{j}(M ; G) \simeq H_{j}(\Gamma(M) ; G) \oplus\left(\oplus_{i=1}^{r} H_{j}\left(M_{i} ; G\right) \text { for all } j>0 ;\right. \\
& \text { (2) } \pi_{1}(M) \simeq \pi_{1}(\Gamma(M)) *\left(*_{i=1}^{r} \pi_{1}\left(M_{i}\right)\right) .
\end{aligned}
$$

Of course the graph $\Gamma(M)$ only contributes to homology in dimension 1. Although it is an extended graph, we can compute its fundamental group just as for graphs. To prove this we also need the next proposition.

Proposition 2.5 If $\Gamma$ is a nonempty connected extended graph, then it contains a maximal tree $T$. Then $\pi_{1}(\Gamma)$ is a free group with one generator for each edge (including loops) of $\Gamma$ which do not belong to $T$.

Proof of Proposition 2.5 First, we construct a connected graph $\Gamma^{\prime}$ from $\Gamma$. For each pair of vertices of $\Gamma$ joined by an edge we remove all but one of the edges; as well we remove any loops (edges from a vertex to itself). What remains is a connected graph $\Gamma^{\prime}$. Then such a graph has a maximal connected tree $T$ (by eg Spanier [19, Chapter 2]). This is also a maximal connected tree for $\Gamma$.

There is only one special case which occurs for $Y$-networks, and hence which we must allow. It is a single $S^{1}$ without any vertex. Then we have to artificially introduce a vertex so $S^{1}$ becomes a loop on the vertex. Then the maximal tree is just the introduced vertex. However, we emphasize that we will not count such "artificial vertices" for later numerical relations involving the number of vertices.

Then by repeating the same proof in [19, Chapter 2] as for the fundamental groups of graphs, $\pi_{1}(\Gamma)$ is a free group with one generator for each edge (including loops) of $\Gamma$ which do not belong to $T$.

We obtain as an immediate corollary. 
Corollary 2.6 If $M$ is contractible, then so is each $M_{i}$ contractible, and furthermore, $\Gamma(M)$ is a tree.

Proof of Corollary 2.6 If $M$ is contractible, then $M$ is connected, $\pi_{1}(M)=0$, and $H_{j}(M)=0$ for all $j>0$. Thus, by (2) of Proposition 2.4, $\pi_{1}(\Gamma(M))=0$; thus, $\Gamma(M)$ is a tree. In addition, for each $i$, by the combination of (1) and (2) of Proposition 2.4, $\pi_{1}\left(M_{i}\right)=0$, and $H_{j}\left(M_{i}\right)=0$ for all $j>0$. Also, by definition, each $M_{i}$ is connected. Then by the Hurewicz Theorem, $\pi_{j}\left(M_{i}\right)=0$ for all $j \geq 0$. However, $M_{i}$ is a CW-complex, so by another Theorem of Hurewicz, $M_{i}$ is contractible.

Proof of Proposition 2.4 By our earlier discussion, $M$ is homotopy equivalent to the space $\widehat{M}$ obtained by attaching $M_{i}$ to $M_{j}$ along an edge segment of $M_{i}$ to a smooth sheet of $M_{j}$. However, this edge segment can be homotoped to a point so $M$ is instead homotopy equivalent to the space obtained by attaching an external curve segment $\alpha_{i j}$ from $x_{i j}$ on the edge of $M_{i}$ to a point $y_{i j}$ in a smooth sheet of $M_{j}$. Then from one fixed point $x_{i 0}$ on $M_{i}$ we can choose disjoint curves $\beta_{i j}$ from $x_{i 0}$ to the other points $x_{i j}$. Then we choose a maximal tree $T$ in $\Gamma(M)$ and choose the attaching curves $\alpha_{i j}$ corresponding to the edges of $T$, as well as the curves $\beta_{i j}$ in each $M_{i}$, which give a tree from each $x_{i 0}$. The resulting space $X$ which is the union of these trees and the $M_{i}$ is homotopy equivalent to the pointed union of the $M_{i}$. Hence,

$$
\pi_{1}(X) \simeq *_{i=1}^{r} \pi_{1}\left(M_{i}\right) \quad \text { and } \quad H_{j}(X) \simeq \oplus_{i=1}^{r} H_{j}\left(M_{i}\right) \text { for } j>0 .
$$

Then as in the computation of $\pi_{1}$ of a graph as in [19, Chapter 2], we inductively add one of the remaining edges and show using Seifert-Van Kampen that we are taking a free product with a free group on one generator. After $k$ steps, where $k$ is the number of additional edges (including loops) of $\Gamma(M)$ not in $T$, we obtain that $\pi_{1}(M)$ is isomorphic to the free product of $\pi_{1}(X)$ with a free group on $k$ generators, which is exactly the fundamental group of $\Gamma(M)$.

For the case of homology we instead use Mayer-Vietoris in a similar argument.

Proposition 2.4 is valid for any region $\Omega$. Thus, we have a relation between the topology of $M$, and hence $\Omega$ (as $M$ is a strong deformation retract of $\Omega$ ), and the individual irreducible medial components $M_{i}$ and the top level extended graph $\Gamma(M)$. In the special case that $\Omega$ is contractible, then so also is $M$ and each $M_{i}$, and $\Gamma(M)$ is a directed tree.

Example 2.7 (Knot complement regions) The simplest example of contractible $\Omega$ corresponds to boundary $\mathcal{B} \simeq S^{2}$. A slightly more complicated example would have $\mathcal{B}$ consisting of two components $S^{2}$ and a torus $T^{2}$. In the case that $T^{2}$ belongs to 
the compact region bounded by $S^{2}$ in $\mathbb{R}^{3}$, then $\Omega$ is the region inside $S^{2}$ and outside of the torus $T^{2}$. This is a "knot complement region".

If $K \subset \mathbb{R}^{3}$ is a knot, then the complement $C=\mathbb{R}^{3} \backslash K$ is not a region in our sense. However, we can take a small tube $T_{\varepsilon}$ around the knot and a large sphere $S_{R}^{2}$ containing the tube. Then the region $\Omega=D_{R}^{2} \backslash \operatorname{int}\left(T_{\varepsilon}\right)$ is a compact region with boundary $S_{R}^{2} \cup \partial T_{\varepsilon}$. Also, $\Omega \subset C$ is a strong deformation retract. For a generic knot with $\varepsilon>0$ and $R$ sufficiently large, the Blum medial axis of $\Omega$ will be generic.

To see what form the irreducible medial components $\left\{M_{i}\right\}$ take for such a knot complement region, we note a few results from knot theory. First, by Alexander duality, $H_{i}(\Omega) \simeq \mathbb{Z}$ for $i<3$ and 0 otherwise. Also, $\pi_{1}(C)$ does not split as a free product of nontrivial groups. Hence, by Proposition 2.4 there are just three possibilities: (i) $\pi_{1}(\Gamma(M)) \neq 0$ so all $M_{i}$ are simply connected; or $\Gamma(M)$ is a tree and all but one of the $M_{i}$, say $M_{1}$, are simply connected; and then either (ii) $H_{2}\left(M_{1}\right) \simeq \mathbb{Z}$, or (iii) $H_{2}\left(M_{1}\right)=0$ and for a single other $M_{i}$, say $M_{2}, H_{2}\left(M_{2}\right) \simeq \mathbb{Z}$.

In the first case, we must have $\pi_{1}(C) \simeq \pi_{1}(\Gamma(M)) \simeq \mathbb{Z}$, which implies $K$ is the unknot. In this case, all but one of $M_{i}$, say $M_{1}$, have trivial reduced homology and hence are contractible, while $M_{1}$ is simply connected with 0 reduced homology except for $H_{2}\left(M_{1}\right) \simeq \mathbb{Z}$. Then $\Gamma(M)$ is homotopy equivalent to $S^{1}, M_{1}$ is homotopy equivalent to $S^{2}$, and $M$ (and hence $C$ ) is homotopy equivalent to the pointed union $S^{1} \vee S^{2}$ (which is homotopy equivalent to a standard homotopy model for the complement of the unknot, the torus with a disk attached to one of the generators of $\pi_{1}$ ).

In the second case, $\Gamma(M)$ is a tree and all of the other $M_{i}$ except $M_{1}$ have trivial fundamental group and reduced homology, and hence are contractible. There is then exactly one noncontractible irreducible medial component $M_{1}$, and $C$ is homotopy equivalent to it.

In the third case, all but $M_{1}$ and $M_{2}$ are contractible, $M_{2}$ is homotopy equivalent to $S^{2}$, and $\Gamma(M)$ is a tree. Then $C$ is homotopy equivalent to $M_{1} \vee M_{2} \simeq M_{1} \vee S^{2}$.

In all of these cases, all but one or two $M_{i}$ are contractible and so contribute nothing to the topology of the region but do contribute to its geometric complexity. Next, we shall determine the structure of the $M_{i}$ and relate this to their topology.

\section{Structure of irreducible medial components}

Next, we decompose each irreducible medial component $M_{i}$, which is connected and locally has only $Y$-junction points, edge points, and 6-junction points (but no fin 
points). We may apply the local closure procedure used in Section 1 to cut the sheets of $M_{i}$ along the remaining $Y$-network as in Figure 14. We do this by locally adding closure points to each of the three sheets meeting at a point of the $Y$-network, or to each of the 6 sheets meeting at 6 -junction points. This disconnects the connected components of the set of smooth points of $M_{i}$ from the $Y$-network, turning them into compact connected surfaces with piecewise smooth boundaries. We refer to each such compact surface with boundary $S$ as a medial sheet of $M_{i}$. The data attached to each medial sheet consists of the triple $(g, o, e)$. Here $g$ is the genus of $S$ (after attaching disks to the boundary components), $o=1$ or 0 corresponding to whether $S$ is orientable or nonorientable (a nonorientable surface with at least one boundary component can be embedded in $\mathbb{R}^{3}$ ), and $e$ denotes the number of boundary components of $S$ (ie $S^{1}$ 's) which are medial edge curves of $M$ (keeping in mind that part of the edge might have originally been an essential fin curve).

Then each $M_{i}$ is formed by attaching certain of the boundary components of medial sheets in $M_{i}$ to the $Y$-network $\mathcal{Y}_{i}$ of $M_{i}$, reversing the cutting process above. The $Y$-network $\mathcal{Y}_{i}$ has connected components $\left\{\mathcal{Y}_{i k}\right\}_{k=1}^{c_{i}}$, and we denote the collection of medial sheets of $M_{i}$ by $\left\{S_{i j}\right\}_{j=1}^{S_{i}}$.
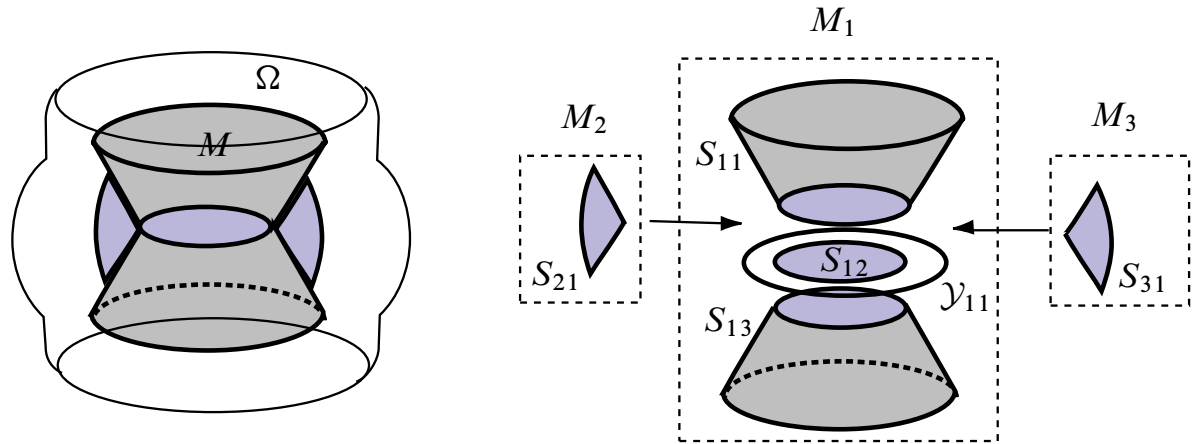

Figure 14: Cutting the medial axis first along fin curves and then along the $Y$-network $\mathcal{Y}$

We assign an extended graph $\Lambda_{i}$ to each such irreducible medial component $M_{i}$ as follows. The graph will have two type of vertices: to each sheet $S_{i j}$ will be associated an $S$-vertex, and to each connected component $\mathcal{Y}_{i k}$ we associate a vertex which we refer to as a $Y$-node. We will associate an edge from the $S$-vertex associated to $S_{i j}$ to the $Y$-node corresponding to $\mathcal{Y}_{i k}$, for each boundary component of $S_{i j}$ (ie an $S^{1}$ ) which is attached to $\mathcal{Y}_{i k}$.

Each medial sheet $S_{i j}$ has the associated data $(g, o, e)$. Second, each connected component $\mathcal{Y}_{i k}$ of the $Y$-network $\mathcal{Y}_{i}$ can be described by an extended graph $\Pi_{i k}$. 
Its vertices correspond to the 6 -junction points of $\mathcal{Y}_{i k}$, and there are distinct edges between vertices which correspond to the distinct $Y$-junction curves of $\mathcal{Y}$ joining the corresponding 6-junction points. Because a 6 -junction point has exactly four $Y$-junction curves ending at it, each vertex of the graph will have four edges. Thus, each vertex will have a valence of 4 , and we will refer to such an extended graph as a "4-valent extended graph".

$M$

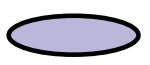

$\mathcal{Y} \varnothing$

$\widetilde{\Pi}$
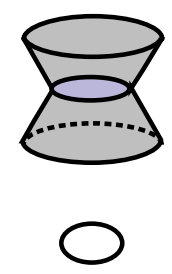

O
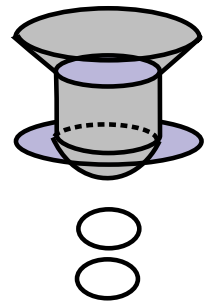

00
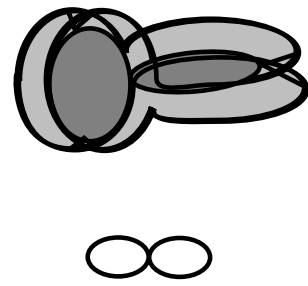

- 4

Figure 15: Simplest examples of irreducible medial components, their $Y_{-}$ networks and the reduced network graphs

A basic result we need for 4-valent extended graphs is a consequence of the following result for $m$-valent extended graphs.

Proposition 3.1 If $\Pi$ is a nonempty connected $m$-valent extended graph with $k$ vertices, then ( $m$ or $k$ must be even and) $\pi_{1}(\Pi)$ is a free group on $\frac{k}{2} \cdot(m-2)+1$ generators.

Hence, for a nonempty connected $4-$ valent graph $\Pi$ with $k$ vertices, $\pi_{1}(\Pi)$ is a free group on $k+1$ generators.

Proof An extended graph $\Pi$ is still a 1 -complex. Hence, $\pi_{1}(\Pi)$ is a free group on $\ell$ generators, where $\ell=\operatorname{rk} H_{1}(\Pi)$. If $\mathrm{V}$, respectively $\mathrm{E}$, denotes the number of vertices, respectively edges, of $\Pi$, then by the $m$-valence of $\Pi, 2 E=m V$. As $V=k$,

$$
\begin{aligned}
\ell & =1-\chi(\Pi)=1-(V-E) \\
& =1-\left(k-\frac{k}{2} \cdot m\right)
\end{aligned}
$$

which equals the value claimed. 


\section{Associated weighted graphs of extended graphs}

In order to work with an extended graph $\Gamma$, we simplify the description by defining an associated weighted graph $\widetilde{\Gamma}$. $\widetilde{\Gamma}$ will have the same vertices as $\Gamma$; however, for each pair of vertices with at least one edge between them, we remove all but one of the edges. Likewise, we remove all loops. Then we assign to each vertex $v_{i}$ the number $2 \ell$ where there are $\ell$ loops at $v_{i}$. Also for the edge between vertices $v_{i}$ and $v_{j}$, we assign the integer $m$ which is the number of edges in $\Gamma$ between $v_{i}$ and $v_{j}$. Then the valence at $v_{i}$ is $2 \ell+\sum m_{i}$ where we sum $m_{i}$ over the edges of $\widetilde{\Gamma}$ which end at $v_{i}$. If for a vertex the number of loops is 0 then we suppress it; likewise if there is only 1 edge between two vertices, we usually suppress the 1 . For extended graph $\Pi_{i k}$ we refer to this graph $\widetilde{\Pi}_{i k}$ as the reduced $Y$-network graph of $\mathcal{Y}_{i k}$. For such a reduced graph, at a vertex the sum of the vertex number and edge numbers always equals 4 . There are very few possibilities for vertices as shown in Figure 16

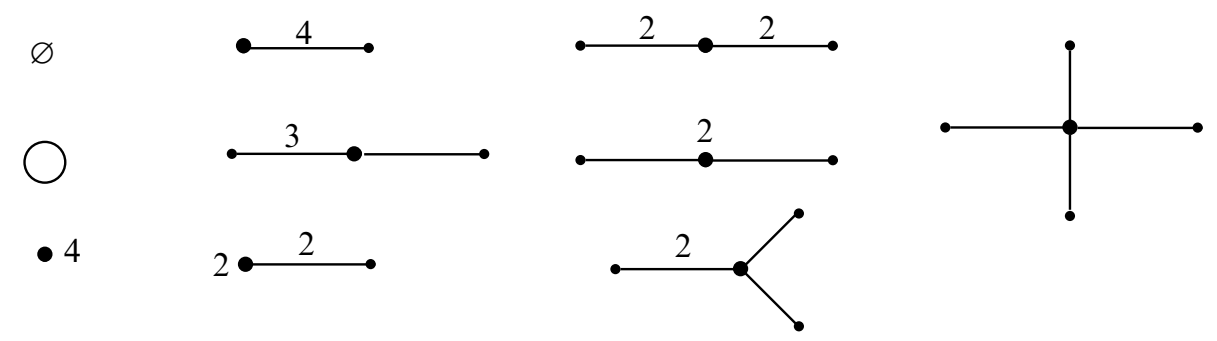

Figure 16: The possible local vertex structures (at the enlarged vertex) for reduced $Y$-network graphs. The case of $S^{1}$ is the exceptional case of nonempty $Y$-network without any vertices.

Finally, we assign this data associated to $M_{i}$ to the graph $\Lambda_{i}$ as follows. The data of $S_{i j}$ will be attached to its $S$-vertex, the graph $\Pi_{i k}$ (or its reduced graph $\widetilde{\Pi}_{i k}$ ) assigned to the $Y$-node of $\mathcal{Y}_{i k}$, and the topological attaching data will be assigned to the edge from $S_{i j}$ to $\mathcal{Y}_{i k}$.

Next, just as we did for $M$, we compute the topological invariants of each irreducible medial component $M_{i}$. This time it will be in terms of the extended graph $\Lambda_{i}$ and the associated data.

\section{Associated numerical data for irreducible medial components}

The numerical invariants can be divided into those that are invariants of the graph $\Lambda_{j}$, and those which are given by the associated data attached to the vertices and edges of $\Lambda_{j}$. In general, for an invariant $I_{j}$ of the medial component $M_{j}$, we let $I=\sum_{j} I_{j}$, summed over the irreducible medial components of $M$, be the associated 
global invariant of $M$. The only exception is our definition of $v(M)$ given by (3-2). We summarize these invariants in Table 1.

Graph-theoretic numerical data of $\Lambda_{j}$ The key invariants associated to $\Lambda_{j}$ are: the number $s_{j}$ of $S$-vertices, the number $c_{j}$ of $Y$-nodes and the first Betti number $\lambda_{j}$ of $\Lambda_{j}$ (as a topological space).

We can express other invariants such as the (reduced) Euler characteristic in terms of these. For example, the number of edges of $\Lambda_{j}=s_{j}+c_{j}+\lambda_{j}-1$. We introduce another purely graph-theoretic invariant which appears in computing $\chi\left(M_{j}\right)$ :

$$
v\left(\Lambda_{j}\right)=s_{j}-c_{j}-\lambda_{j} .
$$

Using this we also introduce the global graph-theoretic invariant of $M$ :

$$
v(M)=\sum_{j} v\left(\Lambda_{j}\right)-\beta_{1}
$$

where the sum is over the irreducible medial components of $M$ and $\beta_{1}$ is first Betti number of $\Gamma(M)$ (as a topological space and $=-\tilde{\chi}(\Gamma(M))$ ).

Numerical associated data of $\Lambda_{j}$ Second, we list the numerical data defined from the associated data. First of all, an $S$-vertex is associated to $S_{j k}$, which is a compact surface with boundary. The boundary will have connected components which are either edge curves or are attached to the $Y$-network $\mathcal{Y}_{j}$. We let $e_{j k}$ denote the number which are medial edge curves of $M_{j}$. For each connected component $\mathcal{Y}_{j k}$ of the $Y$-network, we let $v_{j k}$ denote the number of vertices in $\Pi_{j k}$. This is the number of 6- -junction points in the $Y$-network $\mathcal{Y}_{j}$.

Next, $S_{j k}$ has a genus in the sense that if we attach a 2-disk to each boundary component, then we obtain a compact surface (without boundary). By the classification of surfaces, if it is orientable, it is homeomorphic to a connected sum of tori and the number is its genus; while if the surface is nonorientable, then it is a connected sum of real projective planes, and that number is the genus. In either case we denote the genus by $g_{j k}$, and refer to it as the genus of $S_{j k}$. We define the weighted genus of $S_{j k}$ to be

$$
\tilde{g}_{j k}= \begin{cases}2 g_{j k} & \text { if } S_{j k} \text { is orientable } \\ g_{j k} & \text { if } S_{j k} \text { is nonorientable }\end{cases}
$$

Then the total weighted genus of $M_{j}$

(3-4) $\quad G_{j} \stackrel{\text { def }}{=} \sum_{k} \widetilde{g}_{j k} \quad$ summed over all medial sheets $S_{j k}$ of $M_{j}$. 


\begin{tabular}{|c|c|c|}
\hline $\begin{array}{l}\text { Invariants of } \\
\text { medial components }\end{array}$ & $\begin{array}{l}\text { Global invariants } \\
\text { of medial axis }\end{array}$ & $\begin{array}{l}\text { Description of medial } \\
\text { component invariants }\end{array}$ \\
\hline \multicolumn{3}{|l|}{ Graph invariants } \\
\hline$s_{j}$ & $s$ & $\begin{array}{l}\text { number } S \text {-vertices of } \Lambda_{j}= \\
\text { number medial sheets of } M_{j}\end{array}$ \\
\hline$c_{j}$ & $c$ & $\begin{array}{l}\text { number } Y \text {-nodes of } \Lambda_{j} \\
=\text { number connected } \\
\text { components of } \mathcal{Y}_{j}\end{array}$ \\
\hline$\lambda_{j}$ & $\lambda$ & $\operatorname{rk} H_{1}\left(\Lambda_{j}\right)$ \\
\hline$v\left(M_{j}\right)$ & $v(M)$ & $\begin{array}{l}v\left(M_{j}\right) \text { given by }(3-1) \\
v(M) \text { given by }(3-2)\end{array}$ \\
\hline \multicolumn{3}{|l|}{$\begin{array}{l}\text { Graph data } \\
\text { invariants }\end{array}$} \\
\hline$v_{j}$ & $v$ & $\begin{array}{l}\text { number vertices of } \Pi_{j}= \\
\text { number } 6 \text {-junction points of } M_{j}\end{array}$ \\
\hline$e_{j}$ & $e$ & number medial edge curves of $M_{j}$ \\
\hline$G_{j}$ & $G$ & total weighted genus of $M_{j}(3-4)$ \\
\hline$q_{j}$ & $q$ & sum of $q_{j k}$ given by (3-5) \\
\hline$Q_{j}$ & $Q$ & $\begin{array}{l}\text { first Betti number of associated } \\
\text { 1-complex } \mathcal{S} \mathcal{Y}_{i}^{\prime} \subset M_{j}\end{array}$ \\
\hline
\end{tabular}

Table 1: Invariants $I_{j}$ of Medial Components $M_{j}$ and their Global Versions $I$ for the medial axis, where $I=\sum_{j} I_{j}$.

From the weighted genus, we define $q_{j k}$ as follows.

$$
q_{j k}= \begin{cases}\widetilde{g}_{j k}+e_{j k}-1 & \text { if } e_{j k}>0 \\ \widetilde{g}_{j k} & \text { if } e_{j k}=0\end{cases}
$$

Note this is the first Betti number of a 1-complex in $S_{j k}$ which will be used in Sections 6 to 8 to compute the homology and fundamental group of $M_{j}$. Then we define

$$
q_{j}=\sum q_{j k} \quad \text { summed over all medial sheets } S_{j k} \text { in } M_{j} .
$$

Although $s_{j}$, the number of medial sheets in $M_{j}$, is a graph theoretic invariant, there are subsets of $S$-vertices whose definition depends on associated data. Of these the most important is $s_{0 j}$, the number of sheets without edge curves. This is a sum $s_{0 o, j}+s_{0 n, j}$, 
where $s_{0 o, j}$, resp. $s_{0 n, j}$, denotes the number of orientable, resp. nonorientable, sheets without medial edge curves.

Then by (3-3), (3-4) and (3-5), there is the following relation between these invariants:

$$
q_{j}=G_{j}+e_{j}-\left(s_{j}-s_{0 j}\right)
$$

\section{Topological invariants of irreducible medial components}

We next describe how to determine the Euler characteristic, homology, and fundamental group of $M_{j}$. In Section 8, we introduce a "minimal 1-complex" $\mathcal{S} \mathcal{Y}_{j}^{\prime}$ constructed from the singular data of $M_{j}$. It consists of three contributions: the graph structure $\Lambda_{j}$, the $Y$-network $\mathcal{Y}_{j}$, and a 1 -skeleton from the medial sheets. The $\operatorname{rk} H_{1}\left(\mathcal{S} \mathcal{Y}_{j}^{\prime}\right)$ is given by $Q_{j}=\lambda_{j}+\left(v_{j}+c_{j}\right)+q_{j}$, which is the sum of the three contributions. Then in Section 8, we define an algebraic attaching homomorphism

$$
\Psi_{j}: \mathbb{Z}^{s_{0 j}} \rightarrow \mathbb{Z}^{Q_{j}}
$$

From this homomorphism we can compute the homology of $M_{j}$ in terms of the singular data. Second, we shall also define in Section 7 a set of generators and relations to compute the fundamental group of $M_{j}$. These provide two ingredients for the topology of $M_{j}$.

Theorem 3.2 Suppose that $M_{j}$ is an irreducible medial component. Then there are the following properties in homology:

(1) $M_{j}$ has torsion free homology.

(2) The reduced Euler characteristic of $M_{j}$ is given by

$$
\tilde{\chi}\left(M_{j}\right)=s_{0 j}-Q_{j} .
$$

Alternately, it can be written in terms of the extended graph $\Lambda_{j}$, and associated data of $\Lambda_{j}$ :

$$
\begin{aligned}
\tilde{\chi}\left(M_{j}\right) & =v\left(\Lambda_{j}\right)-\left(G_{j}+e_{j}+v_{j}\right) \\
& =s_{j}-\left(e_{j}+v_{j}+c_{j}+G_{j}+\lambda_{j}\right) .
\end{aligned}
$$

(3) The homology groups $H_{2}\left(M_{j}\right)$, respectively $H_{1}\left(M_{j}\right)$, are the kernel and cokernel of the algebraic attaching homomorphism $\Psi_{j}$; see (8-4). Furthermore, there are bounds

$$
r k\left(H_{2}\left(M_{j} ; \mathbb{Z}\right)\right) \leq s_{0 o, j} \quad \text { and } \quad q_{j} \leq r k\left(H_{1}\left(M_{j} ; \mathbb{Z}\right)\right) \leq Q_{j}-s_{0 n, j} .
$$

In addition, there are the properties for the fundamental group: 
(4) There is a continuous map $\psi_{j}: M_{j} \rightarrow \Lambda_{j}$ such that the induced map $\pi_{1}\left(M_{j}\right) \rightarrow$ $\pi_{1}\left(\Lambda_{j}\right)$ is surjective.

(5) The fundamental group has a presentation by generators and relations

$$
\pi_{1}\left(M_{j}\right) \simeq F_{Q_{j}} /\left\langle\left\{r_{j i}\right\}\right\rangle
$$

where the relations $r_{j i}$ are given by (7-3).

Proof For (1), it is sufficient to show $H_{*}(M)$ is torsion free; for $H_{*}\left(M_{j}\right)$ is a direct summand by Proposition 2.4. Then since $H_{*}(M) \simeq H_{*}\left(\Omega_{0}\right)$ where $\Omega_{0}=\Omega \backslash \mathcal{B}$, it is sufficient to show $H_{*}\left(\Omega_{0}\right)$ is torsion free. We may decompose $\mathbb{R}^{3} \backslash \mathcal{B}=\Omega_{0} \cup \Omega^{\prime}$ as a disjoint union. Thus, $H_{*}\left(\Omega_{0}\right)$ is a direct summand of $H_{*}\left(\mathbb{R}^{3} \backslash \mathcal{B}\right)$. However, by Alexander duality $H_{j}\left(\mathbb{R}^{3} \backslash \mathcal{B}\right) \simeq H^{2-j}(\mathcal{B})$ for $j>0$. Since $\mathcal{B}$ is a compact orientable surface, $H^{*}(\mathcal{B})$ is torsion free, giving the conclusion.

The derivation of the formula for the Euler characteristic, and the computation of the homology in terms of the algebraic attaching homomorphism are given in Section 8.

The computation of the fundamental group is given in Section 7, using the CWdecomposition of $M_{j}$ in terms of the singular structure given in Section 6.

These results provide the following restrictions on geometric complexity.

Corollary 3.3 Consider an irreducible medial component $M_{j}$.

First, suppose $H_{1}\left(M_{j}\right)=0$. Then we have the following:

(1) The graph $\Lambda_{j}$ is a tree.

(2) Each medial sheet $S_{j k}$ has genus 0 , ie it is a 2-disk with a finite number of holes.

(3) At most one of the boundary components of $S_{j k}$ is an edge curve of $M_{j}$.

(4) Suppose, in addition, $H_{2}\left(M_{j}\right)=0$. Then $e_{j}$ is the number of medial sheets with edge curves, and the following "Euler Relation" holds between the number of sheets without edge curves (LHS) and the (RHS), which is rk $H_{1}\left(\mathcal{Y}_{j}\right)$, a topological invariant of the $Y$-network

$$
s_{j}-e_{j}=v_{j}+c_{j} .
$$

(5) If $M_{j}$ is simply connected (without requiring $H_{2}\left(M_{j}\right)=0$ ), then $M_{j}$ will satisfy the fundamental group relation given in Condition 5.1.

(6) Finally, if $M_{j}$ is contractible, then all of the preceding hold. 
The derivation of the Corollary from Theorem 3.2 will be given in Section 8

Finally, we combine the results of Theorem 3.2 with the decomposition in Proposition 2.4 to compute the homology and reduced Euler characteristic of $M$ from the singular invariants measuring geometric complexity. We let

$$
\Psi=\oplus_{j} \Psi_{j}: \mathbb{Z}^{s_{0}} \rightarrow \mathbb{Z}^{Q},
$$

where $s_{0}=\sum_{j} s_{0 j}$ is the total number of medial sheets in all of the $M_{j}$ without edge curves (and $Q=\sum_{j} Q_{j}$ ).

Theorem 3.4 Let $M$ be the Blum medial axis of a connected region $\Omega$ with smooth generic boundary $\mathcal{B}$. Then $H_{*}(\Omega) \simeq H_{*}(M)$ have the following properties.

(1) $M$ (and $\Omega$ ) have torsion free homology.

(2) $H_{2}(M ; \mathbb{Z}) \simeq \operatorname{ker}(\Psi) \quad$ and $\quad H_{1}(M ; \mathbb{Z}) \simeq \operatorname{coker}(\Psi) \oplus H_{1}(\Gamma(M) ; \mathbb{Z})$.

(3) $r k\left(H_{2}(M ; \mathbb{Z})\right) \leq s_{0 o}$ and $q+\beta_{1} \leq \operatorname{rk}\left(H_{1}(M ; \mathbb{Z})\right) \leq Q-s_{0 n}+\beta_{1}$, where $s_{0 o}$, resp. $s_{0 n}$ denote the number of orientable, resp. nonorientable, medial sheets of $M$ without edge curves and as before $\beta_{1}=\operatorname{rk}\left(H_{1}(\Gamma(M) ; \mathbb{Z})\right)$.

(4) The reduced Euler characteristic is given by

$$
\tilde{\chi}(M)=v(M)-(G+e+v)=s-(e+v+c+G+\beta)
$$

where $\beta=\beta_{1}+\sum_{j} \lambda_{j}$.

(5) In the case $\Omega$ (and hence $M$ ) is contractible, $G=0, \beta=0$, and we have the relation

$$
s-e=c+v .
$$

Proof The proof follows from $M$ being a strong deformation retract of $\Omega$ (see eg [6]), using the sum formula given in Proposition 2.4, and substituting the formulas for $H_{i}\left(M_{j} ; \mathbb{Z}\right)$ and $\tilde{\chi}\left(M_{j}\right)$ given in Theorem 3.2.

Example 3.5 The simplest irreducible medial component $M_{i}$ has $\mathcal{Y}_{i}=\varnothing$. Then $M_{i}$ is a compact connected surface with boundary so $s_{i}=1$ and $e_{i}=$ number of boundary components. The $Y$-network invariants $c_{i}=v_{i}=0$ and the graph $\Lambda_{i}$ consists of a single $S$-vertex so $\lambda_{i}=0$. Then $G_{i}=Q_{i}=q_{i}$ is the rank of $H_{1}\left(M_{i}\right)$, and $v\left(M_{i}\right)=1$. If $e_{i}=0$ then $M_{i}$ must be orientable, $s_{0 i}=1$, and $\Psi_{i}$ is the zero map; while if $e_{i}>0$ then $s_{0 i}=0$ and $\Psi_{i}$ is again the zero map. 
Example 3.6 The next simplest case has $\mathcal{Y}_{i}=0$. Already there arises a question of which nonintersecting embedded surfaces can be the medial sheets. The easiest case is when the medial sheets are 2-disks and annuli (examples in Figure 2, Figure 14 and the first three examples of Figure 15). However, an example exists of a single medial sheet which is annulus with a single medial edge curve to form a "collapsed version of the umbilic bracelet" as in Figure 17.

\section{General structure theorem}

We can now state the full structure theorem for the Blum medial axis. and in the next section state the form it takes for contractible $\Omega$.

Theorem 4.1 (General structure theorem) Suppose $\Omega \subset \mathbb{R}^{3}$ is a compact connected region with generic smooth boundary $\mathcal{B}$ and Blum medial axis $M$. Then there is associated to $\Omega$ a two level graph structure.

(1) At the top level $\Gamma(M)$ is a directed extended graph. It consists of vertices corresponding to the irreducible medial components $M_{i}$ of $M$ and directed edges from $M_{i}$ to $M_{j}$ corresponding to each connection attaching an edge of $M_{i}$ to $M_{j}$ along a fin curve.

(2) To each $M_{j}$ is associated a graph $\Lambda_{j}$ with two types of vertices: $S$-vertices corresponding to smooth medial sheets $S_{j k}$ of $M_{j}$, and nodes $\bullet$ corresponding to the connected components $\mathcal{Y}_{j k^{\prime}}$ of the $Y$-network of $M_{j}$. There is an edge from $S_{j k}$ to $\mathcal{Y}_{j k^{\prime}}$ for each boundary $S^{1}$ of $S_{j k}$ which is attached to $\mathcal{Y}_{j k^{\prime}}$.

(3) Each medial sheet $S_{j k}$ is compact surface with boundary (possibly nonorientable). Each $Y$-network component $\mathcal{Y}_{j k^{\prime}}$ can be described as a 4-valent extended graph $\Pi_{j k^{\prime}}$ (or by the corresponding reduced graph $\widetilde{\Pi}_{j k^{\prime}}$ ).

(4) At the third level, the extended graph $\Lambda_{j}$ has data assigned to the $S$-vertices, $Y$-nodes, and edges. To an $S$-vertex for $S_{j k}$ is assigned the data $(g, o, e)$ indicating the genus $g, o=1$ or 0 denoting orientability or nonorientability and $e$ the number of Blum edge curves. To a $Y$-node for $\mathcal{Y}_{j k^{\prime}}$ is assigned the 4 -valent extended graph $\Pi_{j k^{\prime}}$. To an edge from $S_{j k}$ to $\mathcal{Y}_{j k^{\prime}}$, the topological attaching data of the boundary circle of $S_{j k}$ to $\mathcal{Y}_{j k^{\prime}}$.

(5) Furthermore, the graph theoretic data of each $\Lambda_{j}$, the number of edge curves, $6-j u n c t i o n$ points and the weighted total genus satisfy the equation (3-7).

(6) The fundamental groups of each irreducible medial component are given in terms of generators and relations by (7-2), and the homology groups of each irreducible medial component are torsion free and given as the kernel and cokernel of the algebraic attaching map (8-4). 
Proof We have established the decomposition into irreducible medial components, which in turn can be represented by the attaching of medial sheets to the $Y$-network.

The computation of the fundamental group in terms of generators and relations will be given in Section 7 based on the CW-decomposition given in Section 6, and the computation of both the homology and the reduced Euler characteristic will be given in Section 8.

Several examples we have already seen illustrate the general structure theorem.

Example 4.2 In Figure 11, $M$ is formed from 4 medial components, of which $M_{2}$, $M_{3}$, and $M_{4}$ are topologically 2-disks $\left(\mathcal{Y}=\varnothing\right.$ so $c_{i}=v_{i}=0$, and $\left.s_{i}=e_{i}=1\right)$. If we slide $M_{4}$ onto $M_{1}$, then the graph $\Gamma(M)$ is a tree. $M_{1}$ is formed from 5 medial sheets, all of genus 0 : two are 2-disks, 2 are annuli, and one is a 2-disk with two holes. Except for the annuli, each of the other 3 medial sheets of $M_{1}$ have single medial edge curves. The $Y$-network is empty except for $M_{1}$ where it consists of 2 disjoint $\circ$. $\Lambda_{1}$ is a tree, and the attaching of the 2-disks for $M_{1}$ kill the two free generators for $\pi_{1}\left(\mathcal{Y}_{1}\right) \cup \Lambda_{1}$. Hence, $M_{1}$ is simply- connected. Also, we see $v\left(M_{1}\right)=5-2-0=3$ and the other $v\left(M_{i}\right)=1$. Hence, $\widetilde{\chi}(M)=(3+3 \cdot 1+0)-(0+3+3 \cdot 1+0)=0$.

Thus, $H_{2}\left(M_{1}\right)=0$, and $M_{1}$ and hence all of the $M_{i}$ and $M$ are contractible.

Example 4.3 The medial axis shown in Figure 18 is irreducible so $M=M_{1}$. There are five medial sheets $(s=5)$ all of which have genus $0: 3$ annuli (two of which have a medial edge curve) and 2 disks (so $G=0, e=2, q=-1$ and the $Y$-network consists of 2 disjoint $\circ(c=2, v=0)$. Also, $\Lambda_{j}$ is a tree so $\lambda=0$. As above, the attaching of the 2- disks for $M_{1}$ kill the two free generators for $\pi_{1}\left(\mathcal{Y}_{1}\right) \cup \Lambda_{1}$ so $M=M_{1}$ is simply connected. We see $v(M)=5-2+0=3$. Thus, $\tilde{\chi}(M)=3-(0+2+0)=1$. Hence, $H_{2}\left(M_{1}\right)=\mathbb{Z}$, and $M=M_{1}$ is homotopy equivalent to $S^{2}$.

\section{Structure theorem for contractible regions}

For contractible regions $\Omega$, the structure theorem for the Blum medial axis takes a considerably simplified form. We saw in Corollary 3.3 that the medial sheets and extended graphs had to have a special form. As well, we define in Section 7 a set of elements $r_{i j}$ in $\pi_{1}\left(\mathcal{S} \mathcal{Y}_{i}^{\prime}\right)$ corresponding to medial sheets $S_{i j}$ without edge curves; see (7-3). The computation of the fundamental group in Theorem 7.4 leads to the following condition. 
Fundamental Group Condition 5.1 For an irreducible medial component $M_{i}$, the set of elements

$\left\{r_{i j}:\right.$ all $j$ for which $S_{i j}$ is without edge curves of $\left.M_{i}\right\}$

form a set of generators for $\pi_{1}\left(\mathcal{S \mathcal { Y } _ { i } ^ { \prime }}\right)$.

Taken together the conditions on the extended graphs being trees, the numerical "Euler Relation" and the preceding Condition 5.1 completely characterize contractible regions.

Theorem 5.2 (Structure theorem for contractible regions) Suppose $\Omega \subset \mathbb{R}^{3}$ is a contractible bounded region with generic smooth boundary $\mathcal{B}$ and Blum medial axis $M$. Then there is associated to $\Omega$ a multilevel directed tree structure which determines the simplified structure $\hat{M}$ associated to $M$.

(1) At the top level, $\Gamma(M)$ is a directed tree consisting of vertices corresponding to the irreducible medial components $M_{i}$ of $M$, and there are directed edges from $M_{i}$ to $M_{j}$ corresponding to the attaching of an edge of $M_{i}$ to $M_{j}$ along a fin curve in $\hat{M}$.

(2) At the second level, to each $M_{j}$ is associated a directed tree $\Lambda_{j}$ with two types of vertices: $S$-vertices $\mathbf{a}$ corresponding to smooth medial sheets $S_{j k}$ of $M_{j}$, and $Y$-nodes $\bullet$ corresponding to the connected components $\mathcal{Y}_{j k^{\prime}}$ of the $Y$-network of $M_{i}$. There is an edge from $S_{j k}$ to $\mathcal{Y}_{j k^{\prime}}$ if a boundary "circle" of $S_{j k}$ is attached to $\mathcal{Y}_{j k^{\prime}}$. Given $S_{j k}$ and $\mathcal{Y}_{j k^{\prime}}$, there is at most one such boundary "circle".

(3) Each medial sheet $S_{j k}$ is topologically a 2-disk with a finite number of holes. At most one of the boundary circles of a medial sheet is an edge curve of the $M_{j}$. Each $Y$-network component $\mathcal{Y}_{j k^{\prime}}$ can be described as a 4-valent extended graph $\Pi_{j k^{\prime}}$ (or by the corresponding reduced graph $\widetilde{\Pi}_{j k^{\prime}}$.

(4) At the third level, the tree $\Lambda_{j}$ has data assigned to the $S$-vertices, $Y$-nodes, and edges. To an $S$-vertex for $S_{j k}$ is assigned $(h, e)$ indicating the number of holes and Blum edge curves. To a $Y$-node for $\mathcal{Y}_{j k^{\prime}}$ is assigned the 4 -valent extended graph $\Pi_{j k^{\prime}}$. To an edge from $S_{j k}$ to $\mathcal{Y}_{j k^{\prime}}$, the topological attaching data of the single boundary circle of $S_{j k}$ to $\mathcal{Y}_{j k^{\prime}}$.

(5) Furthermore, for an irreducible medial component $M_{j}$, the number of medial sheets, edge curves, 6-junction points and the total number of connected components of the $Y$-network $\mathcal{Y}_{i}$ satisfy the Euler relation (3-8), and each $M_{j}$ satisfies the fundamental group relation Condition 5.1. 
Conversely, suppose we are given a bounded region $\Omega$ in $\mathbb{R}^{3}$ with smooth generic boundary and Blum medial axis $M$ so that: the top level graph $\Gamma(M)$ is a tree; for each irreducible medial component $M_{j}$, the graph $\Lambda_{j}$ is a tree; the medial sheets are topologically 2-disks with a finite number of holes, having at most one boundary circle an edge curve of $M_{j}$; the numerical invariants of $M_{j}$ satisfy (3-8); and each $M_{j}$ satisfies Condition 5.1. Then $M$ and (hence) $\Omega$ are contractible.

Proof If $\Omega$ is contractible, then so is $M$ which is a strong deformation retract of $\Omega$. Then by Corollary 2.6, $\Gamma(M)$ is a tree and each $M_{j}$ is contractible. Then by Corollary 3.3, each $\Lambda_{j}$ is a tree, and each medial sheet $S_{j k}$ is topologically a 2-disk (ie has genus 0 ), with a finite number of holes. At most one of the boundary circles of a medial sheet is an edge curve of the $M$.

Furthermore, $\tilde{\chi}\left(M_{j}\right)=0$, yielding the Euler relation (3-8). As $\pi_{1}\left(M_{j}\right)=0$, by Theorem 7.4, Condition 5.1 must hold.

Conversely, by Theorem 7.4, Condition 5.1 implies that $\pi_{1}\left(M_{j}\right)=0$ for each $j$. Hence, $H_{1}\left(M_{j} ; \mathbb{Z}\right)=0$. As the graphs $\Lambda_{j}$ are trees, $\lambda_{j}=0$, and all sheets have $q_{j}=0$. Thus, the Euler condition implies by (3-7) that $\tilde{\chi}\left(M_{j}\right)=0$. Hence, as $H_{2}\left(M_{j} ; \mathbb{Z}\right)$ is torsion free, $H_{2}\left(M_{j} ; \mathbb{Z}\right)=0$. Thus, as $\Gamma(M)$ is a tree, by Proposition $2.4, M$ is simply connected and $\widetilde{H}_{*}(M ; \mathbb{Z})=0$. As $M$ is a CW-complex, a theorem of Hurewicz implies $M$ is contractible.

Example 5.3 (Simplest examples) As the simplest examples illustrating the structure theorem, we consider those for which the second level trees are $\Pi_{j}=\varnothing$ or $\circ$.

(1) "Simple contractible examples" Each $\Pi_{j}=\varnothing$ so each $M_{i}$ is topologically a 2 -disk. This is the simplest type of region. For example, in Figure 9 and Figure 10, the medial axis is decomposed into irreducible medial components of this type. In computer imaging, the $M$-rep structure of Pizer and coauthors [17] is based on the region having such a simple medial structure.

(2) The second type of regions would allow both $\Pi_{j}=\varnothing$ or $\circ$ and the only type of medial sheets are 2-disks and annuli. Besides the example in Figure 2, we also see the example in Figure 14 and the first three examples of Figure 15 are of this type

(3) Figure 17 is a collapsed version of the "umbilic torus" investigated independently by Helaman Ferguson (who has produced sculptures of it) and Christopher Zeeman who called it the "umbilic bracelet". The discriminant for real cubic binary forms is a cone on this space. The "umbilic torus/bracelet" is obtained by rotating the hypocycloid of three cusps about an external axis in its plane, while rotating by $\frac{2 \pi}{3}$. If we collapse the hypocycloid onto the three line skeleton as in Figure 17 (a), and rotate then we 
obtain Figure 17 (b). It consists of an annulus attached to an inner $Y$-junction curve and an edge curve. It has $\Pi_{j}=\circ$ and a single edge curve, and illustrates how only a single medial sheet may meet the $Y$-junction curve.

(4) The next case would have a reduced network graph of the form "• 4 " as for the fourth example of Figure 15, which is contractible.

It appears to be a rather difficult question to determine exactly when examples such as (3) or (4) can be included as part of larger medial structures with special properties such as being contractible.

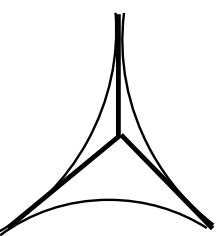

(a) (b)

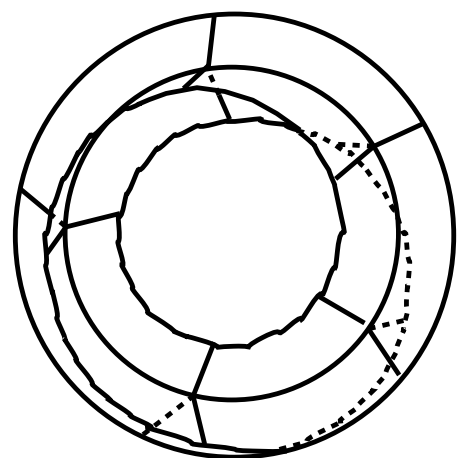

Figure 17: (b) Skeleton of an "umbilic torus/bracelet", obtained by rotating the three intersecting lines in (a) around an axis while rotating by $\frac{2 \pi}{3}$

Example 5.4 (Tree structure for a noncontractible region) A region can have tree structures at each of the two levels and consist of medial sheets of genus 0 attached as prescribed by Theorem 5.2 yet not be contractible. An example is given by Figure 18 . $M$ is not contractible, and it is only the Euler relation that fails: $s=5, e=2$, while $m=2$ and $v=0$, so $s-e=3 \neq 2=v+m$.

\section{Representations of the topological structure of the medial sheets}

To determine the topology of an irreducible medial component $M_{j}$, we give two alternate representations of it as either built up by attaching surfaces with boundaries or by attaching cells. These give two alternate useful ways to decompose $M_{i}$ to compute homology and the fundamental group. We introduce two subspaces $\mathcal{S} \mathcal{Y}_{j} \subset \mathcal{S} \mathcal{Y}_{j}^{\prime} \subset M_{j}$, such that $\mathcal{S} \mathcal{Y}_{j}$ will contain both $\mathcal{Y}_{j}$ and a subspace homotopy equivalent to the extended graph $\Lambda_{j}$. 


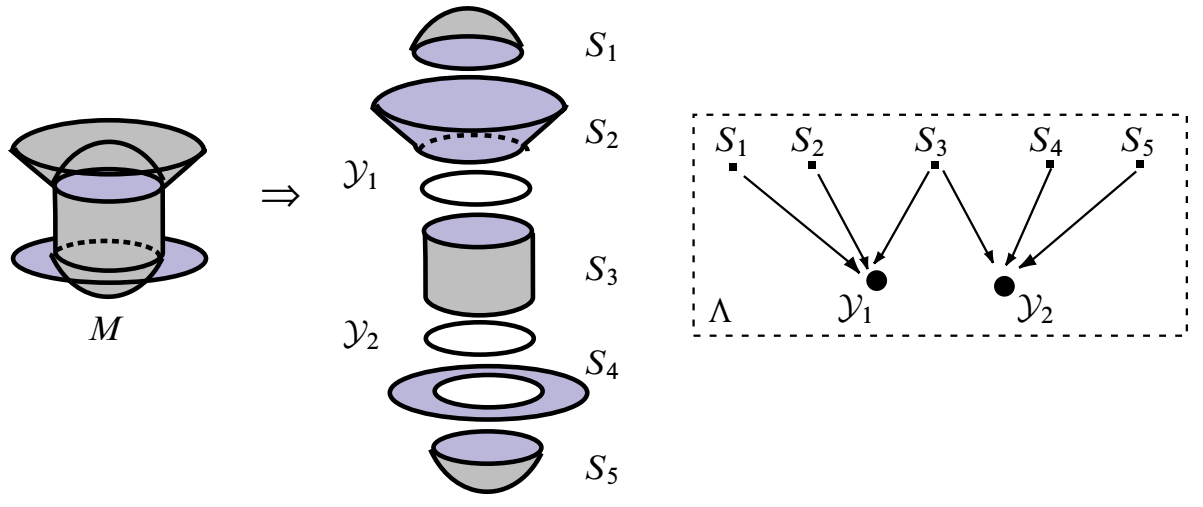

Figure 18: Medial axis $M$ of a noncontractible region with tree structures for $\Gamma(M)$ and $\Lambda$

To do this we consider a medial sheet $S_{j k}$ of $M_{j}$. We may use the standard representation for compact surfaces as quotients of the 2-disk $D^{2}$ after appropriately identifying edges of the boundary as in Massey [15]. In our case, $S_{j k}$ is a quotient of a 2-disk $D^{2}$ with a finite number of holes obtained by removing the interiors $B_{i}$ of embedded 2-disks $\bar{B}_{i}, i=1, \ldots \ell_{j k}+e_{j k}$. Here $\ell_{j k}$ is the number of the disk boundaries which will be attached to $\mathcal{Y}_{j}$, and the other $e_{j k}$ will remain edge curves in $M_{j}$. We may assume that for example, the edges of the boundary of $D^{2}$ are identified as given by the classification of surfaces; see eg Figure 19 (a).

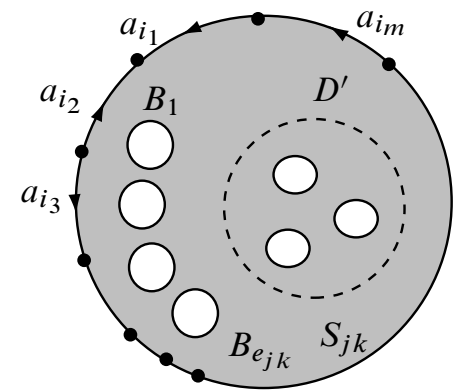

(a)

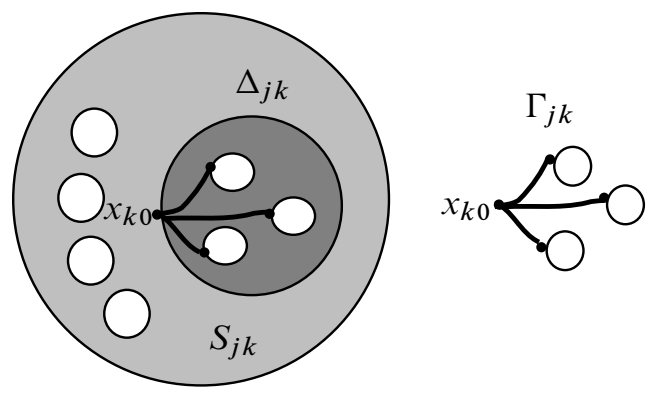

Figure 19: (a) $S_{j k}$ identified as the quotient of a 2-disk with a finite number of holes by identifying the edges of the boundary; (b) Edges of holes to be attached to $\mathcal{Y}$ contained in $\Delta$, and deformation retract $\Gamma_{j k}$.

Thus, $S_{j k}=\left(D^{2} \backslash\left(\bigcup_{i} B_{i}\right)\right) / \sim$. To be specific, we suppose that the boundaries of $\bar{B}_{i}, i=1, \ldots \ell_{j k}$ are the ones which will be attached to $\mathcal{Y}_{j}$. Up to isotopy, we may assume that $\bar{B}_{i}, i=1, \ldots \ell_{j k}$ are contained in a 2-disk $D^{\prime}$ as shown in Figure $19(\mathrm{~b})$. 
We let $\Delta_{j k}=D^{\prime} \backslash\left(\bigcup_{i=1}^{\ell_{j k}} B_{i}\right)$. We may construct curves $\beta_{i}$ in $\Delta_{j k}$ from a point $x_{j 0}$ on the boundary of $\Delta_{j k}$ to boundary points $x_{j i}$ of each $B_{i}$, so the curves are disjoint from each other except at $x_{j 0}$.

We let $\Gamma_{j k}$ denote the union of the curves $\beta_{i}$ and the union of the boundary edges $\partial \bar{B}_{i}, i=1, \ldots \ell_{j k}$. Then $\Gamma_{j k}$ is a strong deformation retract of $\Delta_{j k}$. We let

$$
\mathcal{S} \mathcal{Y}_{j}=\left(\mathcal{Y}_{j} \cup\left(\bigcup_{k} \Delta_{j k}\right)\right) / \sim \quad \text { and } \Gamma_{j}=\left(\bigcup_{k} \Gamma_{j k}\right) / \sim
$$

where $\sim$ denotes the equivalence for attaching the interior boundary edges in the $\Gamma_{j k}$ to $\mathcal{Y}_{j}$. Since every edge of $\mathcal{Y}_{j}$ is the image of one of the attached edges of some $S_{j k}$, we see that $\mathcal{Y}_{j} \subseteq \Gamma_{j} \subset \mathcal{S} \mathcal{Y}_{j}$. Also, $\Gamma_{j}$ is a strong deformation retract of $\mathcal{S} \mathcal{Y}_{j}$ by retracting each $\Delta_{j k}$ to $\Gamma_{j k}$ leaving $\mathcal{Y}_{j}$ fixed.

We next let

$$
\tilde{S}_{j k}=S_{j k} \backslash \operatorname{int}\left(\Delta_{j k}\right) .
$$

Then we can attach each $\widetilde{S}_{j k}$ to $\mathcal{S} \mathcal{Y}_{j}$ along $C_{j k}$ the outer boundary edge of $\Delta_{j k}$. Attaching all such $\widetilde{S}_{j k}$ recovers $M_{j}$. We summarize these statements with the following lemma.

Proposition 6.1 Let $M_{j}$ be an irreducible medial component. Then the following hold:

(1) There is a subspace $\Gamma_{j} \subset \mathcal{S} \mathcal{Y}_{j}$ of $M_{j}$ which contains the $Y$-network $\mathcal{Y}_{j}$.

(2) $\Gamma_{j}$ is a 1-complex which is a strong deformation retract of $\mathcal{S} \mathcal{Y}_{j}$.

(3) If we collapse the connected components of $\mathcal{Y}_{j}$ in $\Gamma_{j}$ to points, then the resulting quotient space is homeomorphic to the second level extended graph $\Lambda_{j}$.

(4) There are compact surfaces with boundaries $\tilde{S}_{j k}$ obtained from the medial sheets $S_{j k}$ by removing $\Delta_{j k}$, a 2 -disk with holes whose edges are identified to $\mathcal{Y}_{j}$. Attaching the $\tilde{S}_{j k}$ to $\mathcal{S} \mathcal{Y}_{j}$ recovers $M_{j}$, up to homeomorphism.

We shall refer to this as the modified medial sheet representation.

\section{CW-decomposition of $M_{j}$}

We further simplify the preceding, by introducing an intermediate space $\mathcal{S} \mathcal{Y}_{j}^{\prime}$.

We divide the medial sheets into two types: those with edge curves and those without. If $S_{j k}$ has an edge curve, then in the earlier notation it has $e_{j k}>0$ edge curves (each surrounding a hole) in the 2-disk. We denote these by $E_{j k}$. We can choose $e_{j k}-1$ disjoint paths $\tau_{k i}$ from $x_{k 0}$ to points on the distinct edge curves $E_{k i}$ for $i<e_{j k}$. We 
can then form $\widetilde{E}_{k i}=E_{k i} \cup \tau_{k i}$. Likewise, we choose a curve $\tau_{k 0}$ from $x_{k 0}$ to the boundary of $D^{2}$, not intersecting the paths $\tau_{k i}$. We can form loops $\sigma_{j i}$ centered at $x_{k 0}$ by following $\tau_{k i}$, then going around the $i$-th edge curve counterclockwise, and then following $\tau_{k i}$ backwards back to $x_{k 0}$ (see Figure 20).
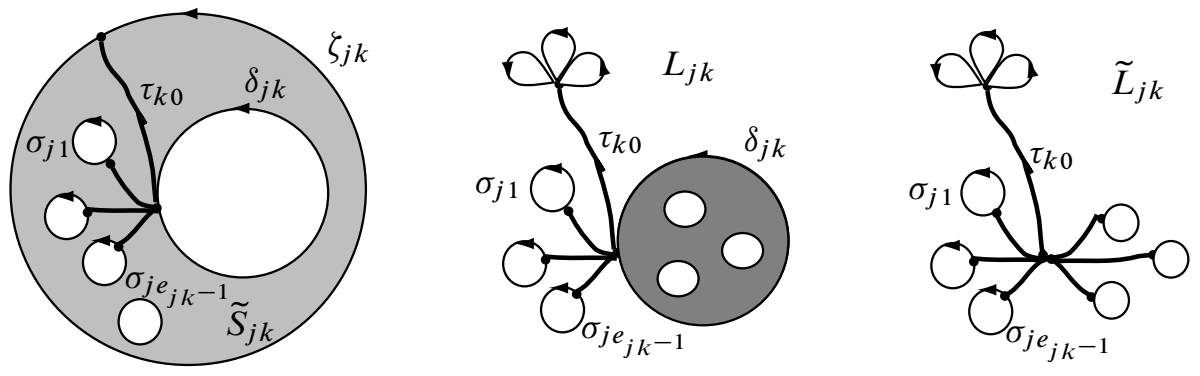

Figure 20: The spaces (a) $\widetilde{S}_{j k}$, (b) $L_{j k}$ and (c) $\tilde{L}_{j k}$

We let

$$
L_{j k}=\Delta_{j k} \underset{k<e_{j k}}{\cup} \widetilde{E}_{j k} \cup \tau_{k 0} \cup\left(\partial D^{2} / \sim\right)
$$

and $\tilde{L}_{j k}$ is defined as $L_{j k}$ except we replace $\Delta_{j k}$ by $\Gamma_{j k}$. Here “ $\sim$ " denotes the identification on the outer boundary of the 2-disk. In the case that $S_{j k}$ has no edge curves of $M_{j}$, then

$$
L_{j k}=\Delta_{j k} \cup \tau_{k 0} \cup\left(\partial D^{2} / \sim\right) .
$$

We define

(6-1) $\mathcal{S} \mathcal{Y}_{j}^{\prime}=\mathcal{S} \mathcal{Y}_{j} \cup_{k} L_{j k}$ where the union is over all medial sheets in $M_{j}$.

We describe the relation between $M_{j}$ and $\mathcal{S} \mathcal{Y}_{j}^{\prime}$ in two steps. First, we relate $S_{j k}$ and $L_{j k}$ (and $\widetilde{L}_{j k}$ ) and then deduce the consequences for $M_{j}$ and $\mathcal{S} \mathcal{Y}_{j}^{\prime}$.

In the case $S_{j k}$ has no edge curves, $S_{j k}$ is homeomorphic to the space obtained by attaching a 2 -disk to $L_{j k}$ as follows.

Lemma 6.2 With the preceding notation:

(1) If $S_{j k}$ has no edge curves, then $S_{j k}$ is homeomorphic to the space obtained by attaching a 2-disk to $L_{j k}$ along the path given in (6-2).

(2) If $S_{j k}$ has edge curves, then $L_{j k}$ is a strong deformation retract of $S_{j k}$.

(3) $\tilde{L}_{j k}$ is a strong deformation retract of $L_{j k}$.

(4) Furthermore, $L_{j k}$ is homotopy equivalent (rel $\Delta_{j k}$ ) to $\Delta_{j k} \vee \bigvee_{i} S^{1}$ (or equivalently $\widetilde{L}_{j k}$ is homotopy equivalent $\left(\mathrm{rel} \Gamma_{j k}\right.$ ) to $\Gamma_{j k} \vee \bigvee_{i} S^{1}$ ), where $\bigvee_{i} S^{1}$ is a bouquet of $q_{j k} S^{1}$ 's (with $q_{j k}$ defined by equation (3-3)). 
Proof If $S_{j k}$ has no edge curves then we proceed as follows to recover $S_{j k}$ from $L_{j k}$ by attaching a $2-$ disk. We cut the annulus $\tilde{S}_{j k}=S_{j k} \backslash \operatorname{int}\left(\Delta_{j k}\right)$ along $\tau_{k 0}$. We obtain a topological 2-disk. Its boundary is mapped to $L_{j k}$ by first following $\tau_{k 0}$, then the boundary of the 2-disk $D^{2}$ in a counter clockwise direction, then backwards along $\tau_{k 0}$ and finally around the boundary $C_{j k}$ of $\Delta_{j k}$ but in a clockwise direction. We denote the counterclockwise path around $\partial D^{2}$ by $\zeta_{j k}^{\prime}$, and the counterclockwise path around the boundary $\Delta_{j k}$ by $\delta_{j k}$. Then a 2-disk is attached along its boundary to $L_{j k}$ by the path

$$
\xi_{j k}=\zeta_{j k} * \bar{\delta}_{j k} \quad \text { where } \quad \zeta_{j k}=\tau_{k 0} * \zeta_{j k}^{\prime} * \bar{\tau}_{k 0}
$$

followed by the identification (as is common, $\bar{\alpha}$ denotes the inverse of a path $\alpha$ ).

Next, suppose instead $S_{j i}$ has $e_{j k}>0$ edge curves. Then we cut $\widetilde{S}_{j k}=S_{j k} \backslash \operatorname{int}\left(\Delta_{j k}\right)$ along the paths $\tau_{k 0}$ and $\tau_{k i}$ for $i<e_{j k}$. The resulting space is topologically an annulus with one boundary the edge curve $E^{\prime} \stackrel{\text { def }}{=} E_{j e_{j k}}$ of the $e_{j k}$-th hole. Then we may retract the annulus onto $L_{j k}$ by retracting along curves from the boundary edge of $E^{\prime}$ as shown, followed by the identification. This gives $L_{j k}$ as a strong deformation retract of $S_{j k}$.

Since this fixes $\Delta_{j k}$, we may then further retract $\Delta_{j k}$ onto $\Gamma_{j k}$, while fixing the rest of $L_{j k}$. This gives $\widetilde{L}_{j k}$ as a strong deformation retract of $L_{j k}$.

Lastly, the boundary of $D^{2}$ after identification is a bouquet of $S^{1}$ 's, where the number is $2 g_{k}$ if $S_{j k}$ is orientable, and $g_{k}$ if $S_{j k}$ is nonorientable.

Finally, returning to $L_{j k}$, if we collapse all of the paths $\tau_{k 0}$ and $\tau_{k i}, i<e_{j k}$, to the point $x_{j 0}$, then $\cup_{i<e_{j k}} E_{j i} \cup \tau_{j} \cup\left(\partial D^{2} / \sim\right)$ becomes a bouquet of $q_{k j} S^{1}$ 's. This collapsing induces a projection map from $L_{j k}$ to $\Delta_{j k} \vee \bigvee_{i=1}^{q_{k j}} S^{1}$ which is the desired homotopy equivalence. An analogous argument works for the space $\widetilde{L}_{j k}$.

Since the deformation retraction for $L_{j k}$ (resp. $\widetilde{L}_{j k}$ ) in (3) of Lemma 6.2 fixes $\Delta_{j k}$ (resp. $\Gamma_{j k}$ ), we may apply the Lemma to each medial sheet with an edge curve after it is attached to $\mathcal{Y}_{j}$. If we let $\mathcal{S} \mathcal{Y}_{j}^{\prime}$ denote $\mathcal{S} \mathcal{Y}_{j}$ with all of these medial sheets attached, then even though each medial sheet $S_{j k}$ contracts to a bouquet of $S^{1}$ 's attached to $\Delta_{j k}$, as $\mathcal{S} \mathcal{Y}_{j}$ is path connected, up to homotopy we may bring these bouquets together and conclude the following "CW-decomposition of $M_{j}$ ".

Proposition 6.3 For an irreducible medial sheet $M_{j}$, we have the following:

(1) $M_{j}$ is homotopy equivalent to a subspace $M_{j}^{\prime}$ obtained from $\mathcal{S} \mathcal{Y}_{j}^{\prime}$ by attaching for each sheet $S_{j k}$ without edge curve a 2-disk along the closed path given by (6-2). 
(2) $\mathcal{S} \mathcal{Y}_{j}^{\prime}$ is homotopy equivalent $\left(\mathrm{rel} \mathcal{S} \mathcal{Y}_{j}\right)$ to $\left(\bigvee_{i=1}^{q_{j}} S^{1}\right) \vee \mathcal{S} \mathcal{Y}_{j}$, and hence to the 1-complex $\left(\bigvee_{i=1}^{q_{j}} S^{1}\right) \vee \Gamma_{j}$ (here $q_{j}$ is defined in Section 3).

Proof By Lemma 6.2, we can first strong deformation retract each sheet $S_{j k}$ with an edge curve onto $L_{j k}$. Then we can obtain each sheet $S_{j k}$ without an edge curve by attaching a 2 -disk to $L_{j k}$. Because this is done while fixing $\mathcal{S} \mathcal{Y}_{j}^{\prime}$, we may do this all at once obtaining the strong deformation retract $M_{j}^{\prime}$.

Also, by Lemma 6.2, each $L_{j k}$ has as a strong deformation retract $\widetilde{L}_{j k}\left(\operatorname{rel} \Gamma_{j k}\right)$, which is homotopy equivalent $\left(\right.$ rel $\left.\Gamma_{j k}\right)$ to $\left(\bigvee_{i=1}^{q_{j k}} S^{1}\right) \vee \Gamma_{j k}$. Again doing this for each $L_{j k}$ gives $\left(\bigvee_{i=1}^{q_{j}} S^{1}\right) \vee \Gamma_{j}$ as a strong deformation retract of $\mathcal{S} \mathcal{Y}_{j}^{\prime}$. Thus, $M_{j}$ is homotopy equivalent to the space obtained from $\left(\bigvee_{i=1}^{q_{j}} S^{1}\right) \vee \Gamma_{j}$ by attaching one 2-disk for each medial sheet $S_{j k}$ without edge curves.

\section{Fundamental group of irreducible medial components}

To compute the fundamental group of an irreducible medial component $M_{j}$, we use the CW-complex decomposition we have just given in Section 6. We successively use the form of the fundamental group for $Y$-network components given in Proposition 3.1 and that of the graph $\Lambda_{j}$ to compute the fundamental group of $\mathcal{S} \mathcal{Y}_{j}$. We then compute the changes obtained by attaching the $L_{j k}$ to give that for $\mathcal{S} \mathcal{Y}_{j}^{\prime}$, and lastly determine the effect of attaching the 2-disks corresponding to $S_{j k}$ without edge curves.

We first compute the fundamental group of $\mathcal{S} \mathcal{Y}_{j}$ as follows.

Proposition 7.1 With the preceding notation,

$$
\pi_{1}\left(\mathcal{S} \mathcal{Y}_{j}\right) \simeq \pi_{1}\left(\Gamma_{j}\right) \simeq \pi_{1}\left(\Lambda_{j}\right) *\left(*_{i=1}^{r} \pi_{1}\left(\mathcal{Y}_{j i}\right)\right)
$$

where there are $r$ components for $\mathcal{Y}_{j}$.

Proof By the preceding remarks, it is sufficient to compute $\pi_{1}\left(\Gamma_{j}\right)$, viewing $\Gamma_{j}$ as an extended graph. We choose a maximal tree $T_{j k}$ for each component $\mathcal{Y}_{j k}$ and a maximal tree $R_{j}$ for $\Lambda_{j}$. Furthermore, as the image of an edge $\partial \bar{B}_{j i}$ will cover completely each edge of $\mathcal{Y}_{j k}$ which it meets, we may choose the points $x_{j k}$ so their images lie in $T_{j k}$, eg they are vertices $y_{m}$.

We also have to treat the special case where one of the $\mathcal{Y}_{j k}$ is an $\circ$ without any vertices. As in the proof of Proposition 2.5, we choose a point in $\circ$ as an artificial vertex, so the $\circ$ can be viewed as a loop on the vertex and then the vertex itself becomes the maximal 
tree. As earlier, we do not count such "vertices" in determining $v_{j}$ for numerical relations.

Then we let $\widetilde{\Gamma}_{j k}$ denote the union of the curves $\beta_{i}$ in $\Gamma_{j k}$ and form

$$
\widetilde{\Gamma}_{j}=\left(\bigcup_{k} \widetilde{\Gamma}_{j k} \cup_{i} T_{j i}\right) / \sim \text {. }
$$

Then each tree $T_{j k}$ is contractible and if we collapse each $T_{j k}$ to a point then the quotient is isomorphic to the extended graph $\Lambda_{j}$.

We may lift the edges of the tree $R_{j}$ to a collection of edges in $\widetilde{\Gamma}_{j}$, by which we mean we can choose curves $\beta_{i}$ in the $\widetilde{\Gamma}_{j k}$ which correspond to the edges of $R_{j}$. We denote the union of those curves by $R_{j}^{\prime}$. Then we form

$$
\mathcal{T}_{j}=\left(R_{j}^{\prime} \cup\left(\bigcup_{i} T_{j i}\right)\right) / \sim
$$

where now “ $\sim$ " only involves attaching the points $x_{j k}$ of $R_{j}^{\prime}$ to the corresponding points $y_{m}$ of the $T_{j i}$.

Lemma 7.2 $\mathcal{T}_{j}$ is a maximal tree in the extended graph $\widetilde{\Gamma}_{j}$.

Proof First, $\mathcal{T}_{j}$ is a tree because each $T_{j i}$ is contractible and if we collapse each to a point we obtain $R_{j}$ which is contractible; hence, $\mathcal{T}_{j}$ is contractible and is a graph.

To see $\mathcal{T}_{j}$ is maximal in $\widetilde{\Gamma}_{j}$, we note any other edge of $\widetilde{\Gamma}_{j}$ either corresponds to an edge of one of the $\mathcal{Y}_{j k}$, or to another edge of $\Lambda_{j}$ under the collapsing map. In the first case, if the edge $\eta$ is in $\mathcal{Y}_{j k}$, then as $T_{j i}$ is maximal $T_{j i} \cup \eta$ is no longer simply connected; thus nor will be $\mathcal{T}_{j} \cup \eta$. If instead the added edge $\eta$ maps to another edge $\eta^{\prime}$ of $\Lambda_{j}$ under the collapsing map, then $R_{j} \cup \eta^{\prime}$ is no longer simply connected. Then we may lift a noncontractible loop $\gamma$ of $R_{j} \cup \eta^{\prime}$ to a loop in $\mathcal{T}_{j} \cup \eta$ (joining edges of $\gamma$ lift to edges which share a common $Y$-node, say $\mathcal{Y}_{j k}$, and hence can be connected by a path in $T_{j i}$ ), destroying the simple connectivity of $\mathcal{T}_{j}$. Thus, $\mathcal{T}_{j}$ is maximal.

Then by Proposition $2.5, \pi_{1}\left(\mathcal{S} \mathcal{Y}_{j}\right)$ is a free group with a free generator for each edge of $\widetilde{\Gamma}_{j}$ not in $\mathcal{T}_{j}$. There are $\lambda_{j}$ edges of $\Lambda_{j}$, and $v_{j k}+1$ edges for each $\mathcal{Y}_{j k}$ by Proposition 3.1. Thus, there are a total of $\lambda_{j}+v_{j}+c_{j}$ free generators (recall $v_{j}=\sum_{k} v_{j k}$ and $c_{j}$ is the number of connected components $\mathcal{Y}_{j k}$ of $\mathcal{Y}_{j}$ ). Such a free group is the free product of the free groups on $v_{j k}+1$ generators which are isomorphic to $\pi_{1}\left(\mathcal{Y}_{j k}\right)$, and a free group on $\lambda_{j}$ generators which is isomorphic to $\pi_{1}\left(\Lambda_{j}\right)$.

The $\pi_{1}\left(\mathcal{Y}_{j k}\right)$ are naturally identified as subgroups of $\pi_{1}\left(\mathcal{S} \mathcal{Y}_{j}\right)$. Also, the collapsing map $\varphi: \widetilde{\Gamma}_{j} \rightarrow \Lambda_{j}$ is a homotopy equivalence; hence, $\pi_{1}\left(\widetilde{\Gamma}_{j}\right) \simeq \pi_{1}\left(\Lambda_{j}\right)$. Then the inclusion $\widetilde{\Gamma}_{j} \subset \mathcal{S} \mathcal{Y}_{j}$, identifies a subgroup isomorphic to $\pi_{1}\left(\Lambda_{j}\right)$. 
The second step is to compute the fundamental group of $\mathcal{S} \mathcal{Y}_{j}^{\prime}$. It is homotopy equivalent to $\left(\bigvee_{i=1}^{q_{j}} S^{1}\right) \vee \mathcal{S} \mathcal{Y}_{j}$. Hence, again by the Seifert-Van Kampen theorem,

$$
\pi_{1}\left(\mathcal{S} \mathcal{Y}_{j}^{\prime}\right) \simeq \pi_{1}\left(\mathcal{S} \mathcal{Y}_{j}\right) * F_{q_{j}}
$$

where $F_{q_{j}}$ is a free group on $q_{j}$ generators corresponding to the generators of $\bigvee_{i=1}^{q_{j}} S^{1}$ obtained from the $E_{j k}$ and the identifications on the boundaries $\partial D^{2} / \sim$. Hence,

Corollary 7.3 $\pi_{1}\left(\mathcal{S} \mathcal{Y}_{j}^{\prime}\right)$ is a free group on $Q_{j}\left(=\lambda_{j}+v_{j}+c_{j}+q_{j}\right)$ generators.

\section{Computing the fundamental group of an irreducible medial component}

We now use the proceeding to compute the fundamental group of $M_{j}$ by determining the effect of attaching each medial sheet $S_{j k}$.

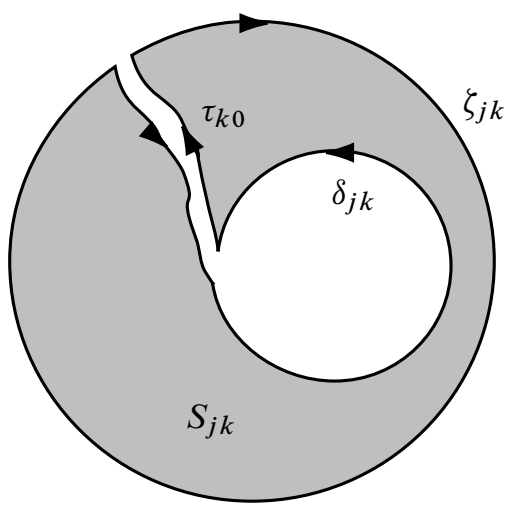

Figure 21: Representing $S_{j k}$ by attaching a 2-disk to $\mathcal{S} \mathcal{Y}_{j}^{\prime}$

Then we can compute the fundamental group of $M_{j}$ by attaching the remaining 2-disks to $\mathcal{S} \mathcal{Y}_{j}^{\prime}$; see Figure 21. First, we recall the loops at $x_{k 0}: \zeta_{j k}$ for each medial sheet without edge curves, and $\delta_{j k}$ (obtained by following $C_{j k}$ in the counterclockwise direction). We again let $\mathcal{T}_{j}$ denote a maximal tree in $\widetilde{\Gamma}_{j}$ and we choose a base point $x_{0} \in \widetilde{\Gamma}_{j}$, and for each $x_{k 0}$, a path $\alpha_{k}$ in the tree $\mathcal{T}_{j}$ from $x_{0}$ to $x_{k 0}$. As $\mathcal{T}_{j}$ is a tree, the path homotopy class of $\alpha_{k}$ only depends on $x_{k 0}$. We form

$$
\tilde{\delta}_{j k}=\bar{\alpha}_{k} * \delta_{j k} * \alpha_{k} \quad \text { and } \quad \tilde{\zeta}_{j k}=\bar{\alpha}_{k} * \zeta_{j k} * \alpha_{k} .
$$

These define elements $r_{j k}=\tilde{\delta}_{j k} * \tilde{\zeta}_{j k}^{-1}$ in $\pi_{1}\left(\mathcal{S} \mathcal{Y}_{j}^{\prime}, x_{0}\right)$, which after composing with the inclusion map yields elements of $\pi_{1}\left(M_{j}, x_{0}\right)$. 
Theorem 7.4 $\pi_{1}\left(M_{j}\right)$ has the representation

$$
\pi_{1}\left(M_{j}\right) \simeq F_{Q_{j}} / N
$$

where $F_{Q_{j}}$ is a free group on $Q_{j}$ generators, and $N$ is the normal subgroup generated by the elements $r_{j k}=\widetilde{\zeta}_{j k} *\left(\widetilde{\delta}_{j k}\right)^{-1}$, one for each medial sheet without edge curves. Equivalently, $\pi_{1}\left(M_{j}\right)$ has exactly the relations

$$
r_{j k}=\tilde{\delta}_{j k} * \tilde{\zeta}_{j k}^{-1}=1 \quad \text { or equivalently } \quad \tilde{\delta}_{j k}=\tilde{\zeta}_{j k} .
$$

Also, for $F_{Q_{j}}, q_{j}$ of the generators correspond to the generators from the identified boundaries of the 2-disks, and $v_{j}+c_{j}$ generators come from $\mathcal{Y}_{j}$.

Also, $\lambda_{j}$ generators come from the generators for $\pi_{1}\left(\Lambda_{j}\right)$. In fact, the collapsing map $\varphi_{j}: \mathcal{S} \mathcal{Y}_{j} \rightarrow \Lambda_{j}$ extends to a continuous map $\tilde{\varphi}_{j}: M_{j} \rightarrow \Lambda_{j}$ which is a surjective map on $\pi_{1}$.

Proof $M_{j}$ is obtained up to homotopy equivalence by attaching 2-disks to $\mathcal{S} \mathcal{Y}_{j}^{\prime}$ along the closed paths given by (6-1); see Figure 21. By the Seifert-Van Kampen theorem this introduces relations killing the corresponding homotopy classes given by (6-1), but with the base point moved to $y_{j}$. These are exactly the relations given in (7-3). The identifications of the various generators from the $\mathcal{Y}_{j k}$, the edge curves of the $S_{j k}$, and the identified boundaries of the 2-disks follow from the Seifert-Van Kampen theorem.

To show that the collapsing map $\varphi_{j}: \mathcal{S} \mathcal{Y}_{j} \rightarrow \Lambda_{j}$ extends to $M_{j}$, we first construct a quotient $\tilde{M}_{j}$ of $M_{j}$ by collapsing the components $\mathcal{Y}_{j k}$ to points. Within $\tilde{M}_{j}$ is the subspace $\widetilde{\mathcal{S}} \mathcal{Y}_{j}$, formed from $\mathcal{S} \mathcal{Y}_{j}$ by collapsing the components $\mathcal{Y}_{j k}$ to points. The collapsing map $\varphi_{j}: \mathcal{S} \mathcal{Y}_{j} \rightarrow \Lambda_{j}$ factors through $\widetilde{\mathcal{S}} \mathcal{Y}_{j}$. Hence, it is sufficient to extend this collapsing map to $\widetilde{M}_{j}$ and then compose with the quotient map $M_{j} \rightarrow \tilde{M}_{j}$.

Then in $\widetilde{\mathcal{S}} \mathcal{Y}_{j}, \Delta_{j k}$ has its inner boundary edges collapsed to points to form $\widetilde{\Delta}_{j k}$, which is topologically a 2 -disk. As a 2 -disk is an absolute retract, we can extend the identity map on $\widetilde{\Delta}_{j k}$ to a continuous map $\widetilde{S}_{j k} \rightarrow \widetilde{\Delta}_{j k}$, where $\widetilde{S}_{j k}$ is $S_{j k}$ with each boundary edge in $\Delta_{j k}$ collapsed to a point. Hence, these continuous maps together give a retract $\tilde{M}_{j} \rightarrow \widetilde{\mathcal{S}} \mathcal{Y}_{j}$. Composed with the collapsing map gives the continuous extension $\tilde{\varphi}_{j}$.

Finally the collapsing map $\widetilde{\Gamma}_{j} \rightarrow \Lambda_{j}$ is a homotopy equivalence, and factors through $\tilde{\varphi}_{j}: M_{j} \rightarrow \Lambda_{j}$. Thus, the induced map on $\tilde{\varphi}_{j *}: \pi_{1}\left(M_{j}\right) \rightarrow \pi_{1}\left(\Lambda_{j}\right)$ is surjective.

Hence, in the special case when $M_{j}$ is simply connected, we obtain Condition 5.1. 


\section{Exact sequence for attaching sheets}

Next, we compute the homology and Euler characteristic of an irreducible medial component $M_{j}$. We use the two ways of obtaining $M$ : by the medial sheet representation, attaching surfaces with boundaries to $\mathcal{S} \mathcal{Y}_{j}$, or the CW-representation obtained by attaching cells to $\mathcal{S} \mathcal{Y}_{j}^{\prime}$. Using these we may compute the exact sequences of pairs $\left(M_{j}, \mathcal{S} \mathcal{Y}_{j}\right)$ and $\left(M_{j}, \mathcal{S} \mathcal{Y}_{j}^{\prime}\right)$. Furthermore, we have the inclusion of pairs $\left(M_{j}, \mathcal{S} \mathcal{Y}_{j}\right) \subset\left(M_{j}, \mathcal{S} \mathcal{Y}_{j}^{\prime}\right)$ This leads to the homomorphism of exact sequences of pairs using reduced homology. Since all spaces have dimension at most 2 the sequence ends at $\mathrm{H}_{2}$.
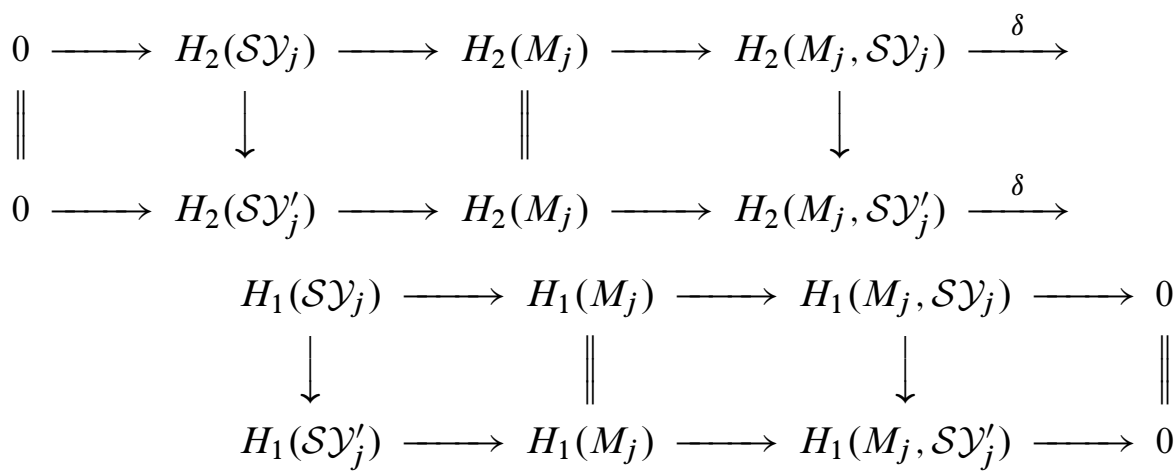

First, using Proposition 6.1 and Proposition 6.3 we can compute the groups $H_{i}\left(\mathcal{S} \mathcal{Y}_{j}\right)$ and $H_{i}\left(\mathcal{S Y} \mathcal{Y}_{j}^{\prime}\right)$, which are zero for $i>1$ and for $i=1$

and

$$
\begin{gathered}
H_{1}\left(\mathcal{S} \mathcal{Y}_{j}\right) \simeq \mathbb{Z}^{\lambda_{j}+v_{j}+c_{j}} \\
H_{1}\left(\mathcal{S} \mathcal{Y}_{j}^{\prime}\right) \simeq \mathbb{Z}^{\lambda_{j}+v_{j}+c_{j}+q_{j}} \simeq \mathbb{Z}^{Q_{j}} .
\end{gathered}
$$

Second, we can compute the relative groups by excision using Proposition 6.1 and Proposition 6.3:

$$
H_{i}\left(M_{j}, \mathcal{S} \mathcal{Y}_{j}\right) \simeq \oplus_{k} H_{i}\left(\tilde{S}_{j k}, C_{j k}\right)
$$

where the sum is over all medial sheets, and

$$
H_{i}\left(M_{j}, \mathcal{S} \mathcal{Y}_{j}^{\prime}\right) \simeq \oplus_{k} H_{i}\left(D^{2}, \partial D^{2}\right)
$$

where the second sum is over medial sheets without edge curves. From this, we conclude $H_{i}\left(M_{j}, \mathcal{S} \mathcal{Y}_{j}^{\prime}\right)=0$ if $i \neq 2$, and

$$
H_{2}\left(M_{j}, \mathcal{S} \mathcal{Y}_{j}^{\prime}\right) \simeq \mathbb{Z}^{s_{0 j}}
$$


(recall $s_{0 j}$ denotes the number of medial sheets of $M_{j}$ which are without edge curves). Likewise, from (8-2), we compute $H_{i}\left(M_{j}, \mathcal{S} \mathcal{Y}_{j}\right)$ using the next lemma.

Lemma 8.1 Suppose $N$ is a compact surface with boundary, with a distinguished boundary component $C$. Let $N^{\prime}$ denote the surface obtained from $N$ by attaching a 2-disk to $N$ along the boundary component $C$. Then

$$
H_{i}(N, C) \simeq \tilde{H}_{i}\left(N^{\prime}\right)
$$

Proof By excision

$$
H_{i}\left(N^{\prime}, D^{2}\right) \simeq H_{i}(N, C) .
$$

Then by the exact sequence of the pair $\left(N^{\prime}, D^{2}\right)$ using reduced homology, we conclude

$$
\tilde{H}_{i}\left(N^{\prime}\right) \simeq H_{i}\left(\tilde{N}, D^{2}\right) .
$$

Hence, by Lemma 8.1:

(1) If $N$ has more than one boundary component, or $N$ is nonorientable, then $\mathrm{H}_{2}(\mathrm{~N}, \mathrm{C})=0$; while if $\mathrm{N}$ is orientable with only the single boundary component $C$, then $H_{2}(N, C)=\mathbb{Z}$.

(2) For $(N, C)=\left(\widetilde{S}_{j k}, C_{j k}\right), \operatorname{rk}\left(H_{1}\left(\widetilde{S}_{j k}, C_{j k}\right)\right)=q_{j k}-\varepsilon_{j k}$, where $\varepsilon_{j k}=1$ if $e_{j k}=0$ and $\widetilde{S}_{j k}$ is nonorientable, otherwise $\varepsilon_{j k}=0$.

Thus, by $(8-2), H_{i}\left(M_{j}, \mathcal{S} \mathcal{Y}_{j}\right)=0$ for $i>2$, and

$$
H_{i}\left(M_{j}, \mathcal{S} \mathcal{Y}_{j}\right) \simeq \begin{cases}\mathbb{Z}^{s_{0 o, j}} & i=2 \\ \mathbb{Z}^{\left(q_{j}-s_{0 n, j}\right)} \oplus\left(\mathbb{Z}_{2}\right)^{s_{0 n, j}} & i=1\end{cases}
$$

where we recall $s_{0 o, j}$, resp. $s_{0 n, j}$, denotes the number of orientable, resp. nonorientable, medial sheets with no edge curves.

Hence, the homomorphism of exact sequences (8-1) takes the form
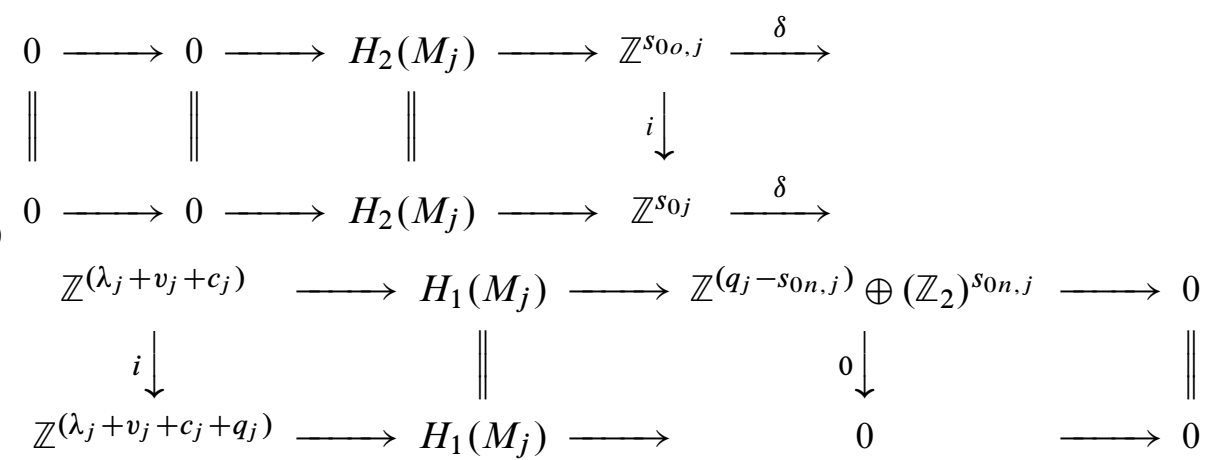
Recall $\mathbb{Z}^{s_{0 j}}=\mathbb{Z}^{s_{0 n, j}} \oplus \mathbb{Z}^{s_{0 o, j}}$ is the number of medial sheets without medial edge curves, and $i$ denotes inclusion of $\mathbb{Z}^{s_{0 o, j}}$.

We can draw a number of conclusions from the diagram (8-3). We let $b_{i}$ denote the $i$-th Betti number of $M_{j}$, and we can take the Euler characteristics of either row and obtain the same formula. For example, from the first row of (8-3),

$$
b_{2}-s_{0 o, j}+\left(\lambda_{j}+v_{j}+c_{j}\right)-b_{1}+\left(q_{j}-s_{0 n, j}\right)=0 .
$$

This yields

$$
\tilde{\chi}\left(M_{j}\right)=s_{0 j}-\left(\lambda_{j}+v_{j}+c_{j}+q_{j}\right)=s_{0 j}-Q_{j} .
$$

Substituting (3-6) into this equation yields (3-7) as asserted.

\section{Computing homology by the algebraic attaching homomorphism}

Next, we use the second row. The middle homomorphism is exactly the algebraic attaching homomorphism

$$
\Psi_{j}: \mathbb{Z}^{s_{0 j}} \stackrel{\delta}{\rightarrow} \mathbb{Z}^{Q_{j}}
$$

It sends the generator of $H_{2}\left(\widetilde{S}_{j k}, C_{j k}\right)$ for orientable $S_{j k}$ without edge curves to the image of its boundary (ie $C_{j k}$ ) under the attaching map to $\mathcal{Y}_{j}$. Here $\mathbb{Z}^{Q_{j}}$ represents the first homology of $\mathcal{S} \mathcal{Y}_{j}^{\prime}$. Then by the exactness of the bottom row of (8-3), the kernel and cokernel of the $\Psi_{j}$ are $H_{2}\left(M_{j}\right)$, respectively $H_{1}\left(M_{j}\right)$, yielding (2) of Theorem 3.2. Furthermore, by the top row of (8-3), we obtain $\operatorname{rk}\left(H_{2}\left(M_{j} ; \mathbb{Z}\right)\right) \leq s_{0 o, j}$. As $H_{*}\left(M_{j} ; \mathbb{Z}\right)$ is torsion free, we can tensor the top row with $\mathbb{Z} / 2 \mathbb{Z}$ and obtain $\operatorname{rk}\left(H_{1}\left(M_{j} ; \mathbb{Z}\right)\right) \geq\left(q_{j}-s_{0 n, j}\right)+s_{0 n, j}=q_{j}$. Also, by the bottom row, or the formula for $\tilde{\chi}\left(M_{j}\right)$,

$$
\operatorname{rk}\left(H_{1}\left(M_{j} ; \mathbb{Z}\right)\right)=Q_{j}-s_{0 j}+b_{2} \leq Q_{j}-s_{0 j}+s_{0 o, j}=Q_{j}-s_{0 n, j} .
$$

This completes the proof of Theorem 3.2

The third consequence of diagram (8-3) is for the cases when various homologies of $M_{j}$ vanish.

Proof of Corollary 3.3 First, suppose $H_{1}\left(M_{j}\right)=0$. From the first row, we conclude that both $q_{j}-s_{0 n, j}=0$ and $s_{0 n, j}=0$. Hence, $q_{j}=s_{0 n, j}=0$. Then $q_{j}=\sum_{k} q_{j k}$ is a sum of nonnegative integers, so $q_{j k}=0$. By (3-3), each $g_{j k}=0$ and if $e_{j k}>0$, then $e_{j k}=1$. In particular, $S_{j k}$ must be a 2 -disk with a finite number of holes, and 
the edge of at most one of the holes is an edge curve of $M_{j}$. In particular, all of the medial sheets are orientable so

$$
s_{0 o, j}=s_{0 j}=s_{j}-e_{j} .
$$

This establishes (2) and (3) of Corollary 3.3. Furthermore, by Theorem 7.4, the collapsing map $p: M_{j} \rightarrow \Lambda_{j}$ induces a surjection on $\pi_{1}$ and hence on the abelianized $\pi_{1}$. Hence, $H_{1}\left(\Lambda_{j} ; \mathbb{Z}\right)=0$, so $\Lambda_{j}$ is a tree and $\lambda_{j}=0$, establishing (1) of Corollary 3.3 .

Second, suppose in addition that $H_{2}\left(M_{j} ; \mathbb{Z}\right)=0$. Then

$$
\delta: \mathbb{Z}^{s_{0 o, j}} \simeq \mathbb{Z}^{\left(\lambda_{j}+v_{j}+c_{j}\right)} .
$$

Thus,

$$
s_{0 o, j}=\lambda_{j}+v_{j}+c_{j} .
$$

Hence, as $\lambda_{j}=0$, this equation and (8-5) together imply

$$
s_{j}-e_{j}=v_{j}+c_{j} .
$$

The remaining conditions on the fundamental group follow from Theorem 7.4.

\section{Algorithm for contracting contractible medial axes}

In the contractible case, the structure suggests a method for contracting a region. We would like a specific algorithm for contracting the medial axis when it is contractible. First, we can deform it by sliding along fin curves so we have the simplified structure $\hat{M}$. Then we want to simplify each irreducible medial component. If the medial component has a nonempty $Y$-network, then we choose a medial sheet with a medial edge curve, and deform the medial sheet so the medial edge curve touches the $Y$-network. At that point a transition occurs with two fin points being created as in Figure 22. We can then slide the fin curve until it doesn't meet the remaining $Y$-network. This does not change the homotopy type of the medial component.

We repeat this process as long as the $Y$-network of the component is nonempty. Applying this to all components, we reach a point where all $Y$-networks are empty. In the process we have created more irreducible components attached to each other along fin curves. By contractibility, all of the medial sheets are 2-disks. The attaching is described by a tree. We then proceed to simplify the tree.

We take one of the outermost branches. It corresponds to a 2-disk attached to another sheet along a segment of its boundary as a fin curve. We contract down the sheet and 

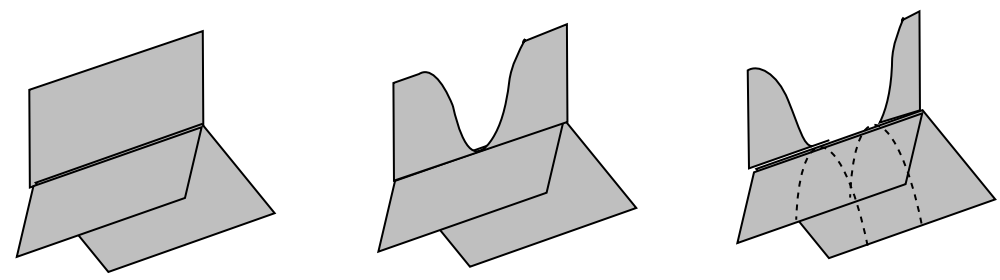

Figure 22: Creation of a pair of fin points from a medial edge curve

the fin curve to a point. Then we repeat this process until there is only a single medial component left. It is a 2 -disk and hence contractible.

The only point about the preceding argument is the assertion that if a medial component is contractible then it has an edge curve. This step has not been completed and we are left with a conjecture.

Conjecture If an "irreducible medial component" is contractible then it has an edge curve.

The "irreducible medial component" is in quotes because after the first step we no longer know that it really comes from the medial axis of a region. What we really are asking is whether a singular space which is embedded in $\mathbb{R}^{3}$ and has the local singular structure of a medial axis satisfies the conjecture.

\section{Discussion and open questions}

There are a number of basic questions which are unanswered.

(1) The conditions we gave are for a medial axis which already exists. Given an abstract model for a medial axis, satisfying all of the properties, this model may not be realized in $\mathbb{R}^{3}$. In particular, we must understand the conditions allowing embeddings of the medial sheets so they do not intersect.

(2) Likewise, there are related restrictions on the attaching maps to $Y$-network so that three sheets are attached at each point. What constraints does this place on possible structures? This and the preceding point appear to involve subtle questions in topology.

(3) In order to complete the algorithm for contracting regions, we need to prove the existence of edge curves on contractible irreducible "medial components". 
(4) The decomposition into irreducible medial components requires operations on fin curves. These local operations (with the exception of the cutting of essential fin curves) are examples of local deformations of medial axes resulting from deformations of regions by results of Giblin and Kimia [12] building on the results of Bogaevsky [2; 3]. Can we obtain the operations on fin curves as global deformations of the region?

(5) The structure of the medial axis for specific types of regions such as, for example, knot complement regions contains topological information about the region. For example, if we look for a topological representative of the region for which the number of medial sheets is minimal, then as the reduced Euler characteristic is fixed, we obtain from (3-7) an expression for $s_{j}-\tilde{\chi}\left(M_{j}\right)$ as a sum of five nonnegative integers $e_{j}+v_{j}+c_{j}+G_{j}+\lambda_{j}$. What possible partitions of $s_{j}-\tilde{\chi}\left(M_{j}\right)$ into five nonnegative integers are actually possible, and how does this relate to the structure of the original knot?

\section{References}

[1] H Blum, R Nagel, Shape description using weighted symmetric axis features, Pattern Recognition 10 (1978) 167-180

[2] I A Bogaevskiŭ, Reconstructions of singularities of minimum functions, and bifurcations of shock waves of the Burgers equation with vanishing viscosity, Algebra i Analiz 1 (1989) 1-16 MR1027457

[3] I A Bogaevsky, Perestroikas of shocks and singularities of minimum functions, Phys. D 173 (2002) 1-28 MR1945478

[4] M A Buchner, The structure of the cut locus in dimension less than or equal to six, Compositio Math. 37 (1978) 103-119 MR0501100

[5] J Damon, Global Geometry of Regions and Boundaries via Skeletal and Medial Integrals, to appear in Comm. Anal. and Geom.

[6] J Damon, Smoothness and geometry of boundaries associated to skeletal structures. I. Sufficient conditions for smoothness, Ann. Inst. Fourier (Grenoble) 53 (2003) 19411985 MR2038785

[7] J Damon, Smoothness and geometry of boundaries associated to skeletal structures. II. Geometry in the Blum case, Compos. Math. 140 (2004) 1657-1674 MR2098407

[8] J Damon, Determining the Geometry of Boundaries of Objects from Medial Data, Int. Jour. Comp. Vision 63 (2005) 45-64

[9] ME Gage, Curve shortening makes convex curves circular, Invent. Math. 76 (1984) 357-364 MR742856 
[10] M E Gage, R S Hamilton, The heat equation shrinking convex plane curves, J. Differential Geom. 23 (1986) 69-96 MR840401

[11] P Giblin, Symmetry sets and medial axes in two and three dimensions, from: "The mathematics of surfaces, IX (Cambridge, 2000)", Springer, London (2000) 306-321 MR1846302

[12] P Giblin, B Kimia, Transitions of the 3D Medial Axis Under a One-Parameter Family of Deformations, from: "Proc. ECCV 2002", Lecture Notes in Computer Science 2351, Springer, London (2002) 718-734

[13] M A Grayson, The heat equation shrinks embedded plane curves to round points, J. Differential Geom. 26 (1987) 285-314 MR906392

[14] B B Kimia, A Tannenbaum, S Zucker, Toward a computational theory of shape: An overview, from: “Three Dimensional Computer Vision”, (O Faugeras, editor), MIT Press, Boston (1990) 402-407

[15] W S Massey, A basic course in algebraic topology, Graduate Texts in Mathematics 127, Springer, New York (1991) MR1095046

[16] J N Mather, Distance from a submanifold in Euclidean space, from: "Singularities, Part 2 (Arcata, CA, 1981)", Proc. Sympos. Pure Math. 40, Amer. Math. Soc., Providence, RI (1983) 199-216 MR713249

[17] S Pizer et al, Deformable M-reps for 3D Medical Image Segmentation, Int. Jour. Comp. Vision 55 (2003) 85-106

[18] J A Sethian, Level set methods, Cambridge Monographs on Applied and Computational Mathematics 3, Cambridge University Press, Cambridge (1996) MR1409367Evolving interfaces in geometry, fluid mechanics, computer vision, and materials science

[19] EH Spanier, Algebraic topology, McGraw-Hill Book Co., New York (1966) MR0210112

[20] Y Yomdin, On the local structure of a generic central set, Compositio Math. 43 (1981) 225-238 MR622449

Department of Mathematics, University of North Carolina

Chapel Hill, NC 27599-3250, USA

jndamon@math. unc.edu

Proposed: Colin Rourke

Received: 2 February 2006

Seconded: Robion Kirby, Walter Neumann

Accepted: 30 August 2006 